\title{
Pion production in inelastic and central nuclear collisions at high energy
}

\author{
M. Anikina, A. Golokhvastov, K. Iovchev, S. Khorozov, E. Kuznetzova, J. Lukstins, \\ E. Okonov, T. Ostanevich, V. Toneev, and G. Vardenga \\ Joint Institute for Nuclear Research, Dubna, Union of Soviet Socialist Republics
}

L. Chkhaidze and T. Dzobava

Tbilisi State University, Tbilisi, Union of Soviet Socialist Republics

M. Gaździcki, E. Skrzypczak, and R. Szwed

University of Warsaw, Warsaw, Poland

\author{
K. Gudima \\ Institute of Applied Physics, Moldavian Academy of Science, Kishinev, Union of Soviet Socialist Republics
}

(Received 3 May 1985)

\begin{abstract}
A detailed study of pion production in inelastic and central nucleus-nucleus collisions was carried out using a $2 \mathrm{~m}$ streamer spectrometer. Nuclear targets mounted inside the streamer chamber were exposed to nuclear beams of $4.5 \mathrm{GeV} / \mathrm{c} /$ nucleon momentum. A systematic study of the influence of the central trigger on observed data is performed. The data on multiplicities, rapidities, transverse momenta, and emission angles of negative pions are presented for various pairs of colliding nuclei. Intercorrelations between various characteristics are studied and discussed. The results are compared with predictions of some theoretical models. It is shown that the main features of the pion production in nuclear collisions can be satisfactorily described by a model assuming independent nucleon-nucleon collisions with subsequent cascading process. However, the observed correlation between $\Lambda$ and pion characteristics seems to be unexplained by this picture.
\end{abstract}

\section{INTRODUCTION}

It has been realized long ago that the analysis of nucleus-nucleus $\left(A_{P}-A_{T}\right)$ collisions at relativistic energies yields a unique possibility of studying properties of dense highly excited nuclear matter. Many experiments were carried out ${ }^{1-3}$ and several more are being planned ${ }^{4}$ in order to test theoretical predictions concerning the processes involved in particle production within the nuclear matter at high energy. Theoretical models predict in particular the formation of exotic states of nuclear matter, e.g., phase transition to quark-gluon plasma. ${ }^{5}$ Energy density $\epsilon$ required for a phase transition to the quark-gluon plasma state is, according to theoretical estimates, $1-2$ $\mathrm{GeV} / \mathrm{fm}^{3}$ (Ref. 5). In the case of the Dubna accelerator (4.5 GeV/c momentum per incident nucleon) a rough estimation of the energy density from the simple formula $\epsilon=2 \gamma_{\text {c.m. }}^{2} \rho_{0} m_{\mathrm{N}}$ (see, e.g., Ref. 6) leads to $\epsilon \approx 1 \mathrm{GeV} / \mathrm{fm}^{3}$. It is expected that the effect of the dynamical compression of the colliding nuclear matter may lead to an increase of the $\epsilon$ value (see Ref. 7 and Fig. 4 therein). Consequently, a transition to the quark-gluon plasma may be expected even at the Dubna energy, especially if the full stopping of the colliding nuclear matter takes place, e.g., in some appropriately chosen classes of events. It seems worthwhile, therefore, to carry out a detailed analysis of the particle production in various classes of nuclear collisions (i.e., to perform a "semiexclusive" analysis).

Our previous results on pion production ${ }^{8-11}$ and on strange particle production in inelastic nucleus-nucleus collisions $^{12,13}$ showed that these particles are produced mainly in independent nucleon-nucleon (N-N) collisions.

In this paper the new results of a detailed analysis of pion production in inelastic and in central nucleus-nucleus collisions are presented. The analysis is based on measurements of negative pions emitted from nuclear collisions. The cross-section values for interactions characterized by a different "degree of centrality" are presented and discussed in Sec. III.

Multiplicity distributions of negative pions and semiinclusive distributions of various kinematical characteristics, like rapidity and transverse momentum, are studied and discussed in Secs. IV and V. A quantitative analysis of the form of the distributions (of any variable $x$ ) is based essentially on a study of the statistical moments of the distributions, namely the average value $(\langle x\rangle)$, dispersion $\left(D_{x}\right)$, and skewness $\left[\gamma_{x}^{1}=\mu_{3} / D_{x}^{3} ; \mu_{3}=\left\langle(x-\langle x\rangle)^{3}\right\rangle\right]$. Detailed numerical data on cross section and on the values of the moments of the distributions characterizing pion production were published previously. ${ }^{14}$ Special attention is paid to subsamples of events, in which baryons $(\Lambda$ hyperons, protons) produced beyond $\mathrm{N}-\mathrm{N}$ kinematical limits have been observed (Sec. VI).

Presentation of our experimental data in figures in this paper is in most cases accompanied by predictions of theoretical models discussed in Sec. VII. This section is devoted to the discussion of obtained experimental results from the point of view of three theoretical approaches, namely: "no-cascading" model, intranuclear cascade 
model, and thermodynamic approach. The experimental results and conclusions are summarized in Sec. VIII.

\section{EXPERIMENTAL}

The data were obtained from pictures of a $2 \mathrm{~m}$ streamer chamber SKM-200 (Refs. 14 and 15) placed in a magnetic field of $0.8 T$ and exposed to beams of nuclei accelerated in the Dubna synchrophasotron up to $4.5 \mathrm{GeV} / c$ momentum per incident nucleon. Solid targets in the form of thin discs were mounted within the chamber. Neon gas filling the chamber served also as a nuclear target. Two triggering systems were used:

(a) minimum bias trigger selecting all inelastic interactions of incident nuclei on a target:

(b) central triggering system selecting interactions without charged fragments of the projectile nucleus $(p / Z \widetilde{>} 3 \mathrm{GeV} / c)$ emitted at angles $\theta<\theta_{\mathrm{ch}}=2.4^{\circ}$ or $2.9^{\circ}$ and without fast neutrons emitted at angles $\theta<\theta_{n}=1.8^{\circ}$ or $2.8^{\circ} .14,15$

The different $\theta_{\mathrm{ch}}$ and $\theta_{n}$ angles used in the experiment resulted from limited space for placing the triggering detectors. The experimental setup and the logic of the triggering systems are presented in Fig. 1. During the scanning two subsamples of central events, corresponding to $\theta_{\mathrm{ch}}=5^{\circ}$ and $14^{\circ}$, were selected.

Since several combinations of $\theta_{\text {ch }}$ and $\theta_{n}$ values have been used in the course of analysis, the trigger mode for each sample of events is denoted hereafter as $T\left(\theta_{\text {ch }}, \theta_{n}\right)(\theta$ values rounded to integers).

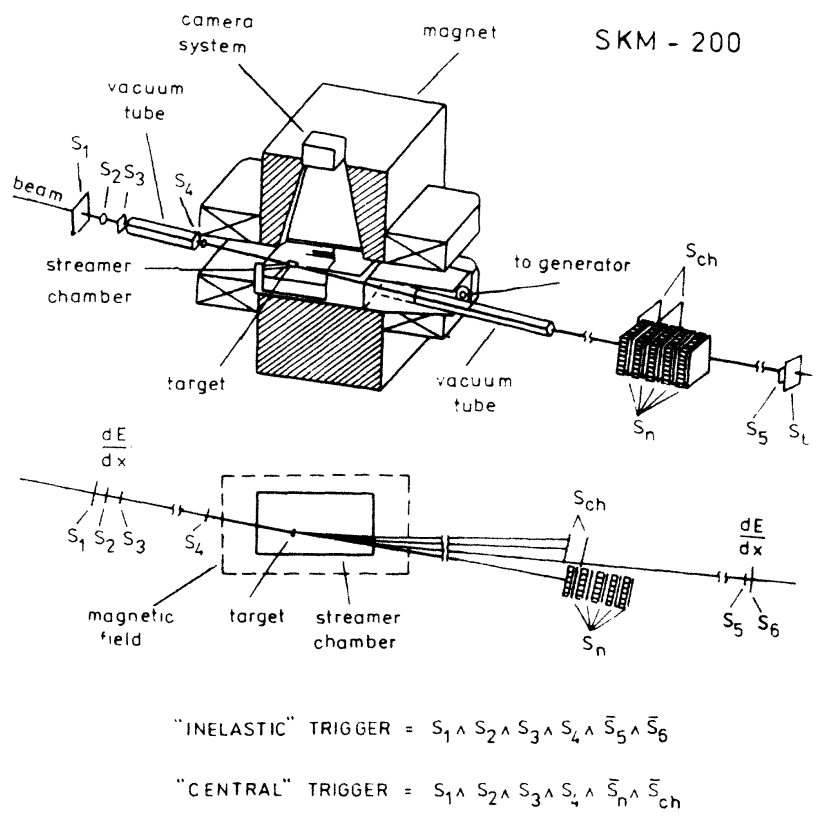

FIG. 1. Experimental setup. The trigger and the trigger distances are not up to scale.
Primary results of scanning and measurements were biased due to several experimental effects and appropriate corrections were introduced. The biases and correction procedures were discussed in detail in Ref. 14. The errors of cross-section values and of the characteristics of multiplicity distributions include statistical and systematical uncertainties. In the case of multiplicity distributions the total uncertainty of the corrections ranged from $3 \%$ to $5 \%$. In the case of pion kinematical characteristics systematic errors do not exceed statistical ones, therefore only statistical errors are presented. Methods of the identification of $\Lambda$ hyperons and separation of protons emitted into the backward hemisphere were described in detail in Refs. 15 and 16.

\section{CROSS SECTIONS}

It is well known that inelastic cross sections for nucleus-nucleus collisions are determined mainly by the geometry of colliding objects. Therefore the data on $\sigma^{\text {inel }}$ are satisfactorily described by a simple "geometrical" formula: ${ }^{17}$

$$
\sigma^{\text {inel }}=\pi r_{0}^{2}\left(A_{P}^{1 / 3}+A_{T}^{1 / 3}-b_{0}\right)^{2},
$$

where $r_{0}$ and $b_{0}$ are fitted parameters.

A more complicated situation arises in case of central collisions. The $\sigma^{\text {cent }}$ values are very sensitive to the experimental definition of a central collision and to trigger biases. ${ }^{14}$ It seems that values of cross sections for central collisions are determined not only by geometrical factors but also by the dynamical effects, such as the transparency of nuclear matter and the depletion of spectator nucleons due to subsequent nucleon-nucleon collisions, especially important for colliding nuclei with close mass numbers $\left(A_{P} \approx A_{T}\right)$.

The dependence of $\sigma$ values on the degree of centrality (determined by $\theta_{\text {ch }}$ and $\theta_{n}$ values) is shown in Fig. 2. In order to present these data independently of the beam energy, instead of the veto angles, the $p_{T}^{\operatorname{ch}(n)}$ values, being maximum transverse momenta of positive secondaries treated by the trigger as projectile fragments, are used $\left[p_{T}^{\operatorname{ch}(n)}=p_{\mathrm{INC}} \tan \left(\theta_{\mathrm{ch}(n)}\right)\right.$, where $\left.p_{\mathrm{INC}}=4.5 \mathrm{GeV} / c\right]$. A rapid decrease of the $\sigma$ is observed for $p_{T}^{\mathrm{ch}} \widetilde{<} 250 \mathrm{MeV} / c$, whereas for $p_{T}^{\mathrm{ch}} \sim 250 \mathrm{MeV} / c$ the decrease of $\sigma$ is much less steep. The momenta of projectile fragments are determined mainly by internal momenta of nucleons, $p_{F}$, within the projectile nuclei. Consequently, the use of $p_{T}^{\mathrm{ch}} \sim p_{F}\left(p_{F}=220 \mathrm{MeV} / c\right.$ for ${ }^{12} \mathrm{C}$ nuclei, ${ }^{18} p_{T}^{\text {ch }}=p_{F}$ corresponds to $\theta_{\mathrm{ch}}=2.8^{\circ}$ ) leads to the rejection of practically all events with charged spectator fragments of projectile nuclei. A further increase of the $p_{T}^{\text {ch }}$ value leads to the selection of subsamples of central events with high inelasticity. One should note that at our energy $99 \%$ of protons from single p-p elastic interactions are emitted at angles less than $14^{\circ}$. The influence of $\theta_{n}$ (in our experiment $\theta_{n}<\theta_{\mathrm{ch}}$ ) on $\sigma$ value is significant only in the case when $A_{P} \approx A_{T}$ and it is rather weak for heavier target nuclei (see the discussion in Sec. IV).

The dependence of $\sigma$ values on $A_{P}$ is shown in Fig. 3 for four trigger modes: $T(0,0), T(2,0), T(5,0)$, and $T(14,0)$. The solid lines in Fig. 3 correspond to the pa- 


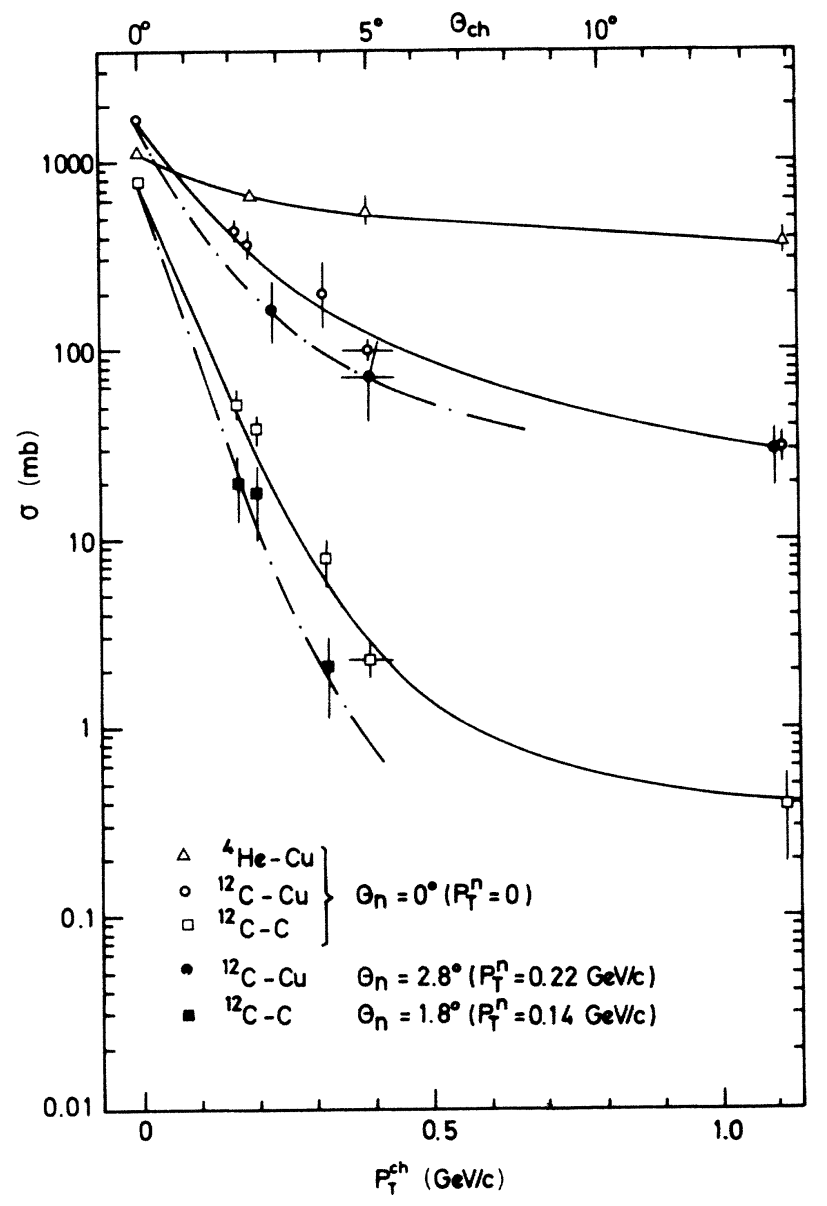

FIG. 2. The dependence of cross section values for inelastic and central nuclear interactions on $p_{T}^{\mathrm{ch}}=p_{\mathrm{INC}} \tan \left(\theta_{\mathrm{ch}}\right)$ for various $p_{T}^{n}=p_{\mathrm{INC}} \tan \left(\theta_{n}\right)$ values $\left[p_{\mathrm{INC}}=4.5 \mathrm{GeV} / c ; \theta_{\mathrm{ch}(n)}\right.$, veto angles for charged (neutral) fragments of projectile nuclei]. Solid (for $\left.\theta_{n}=0\right)$ and dashed-dotted (for $\theta_{n} \neq 0$ ) lines are drawn to guide the eye only.

rametrization of experimental data using the following formulas:

$$
\sigma^{\text {inel }}=\pi r_{0}^{2}\left(A_{P}^{1 / 3}+A_{T}^{1 / 3}-b_{0}\right)^{2}
$$

and

$$
\sigma^{\text {cent }}=C A_{T}^{\alpha}
$$

for inelastic and central collisions, respectively. The fits were performed using all available data on inelastic cross

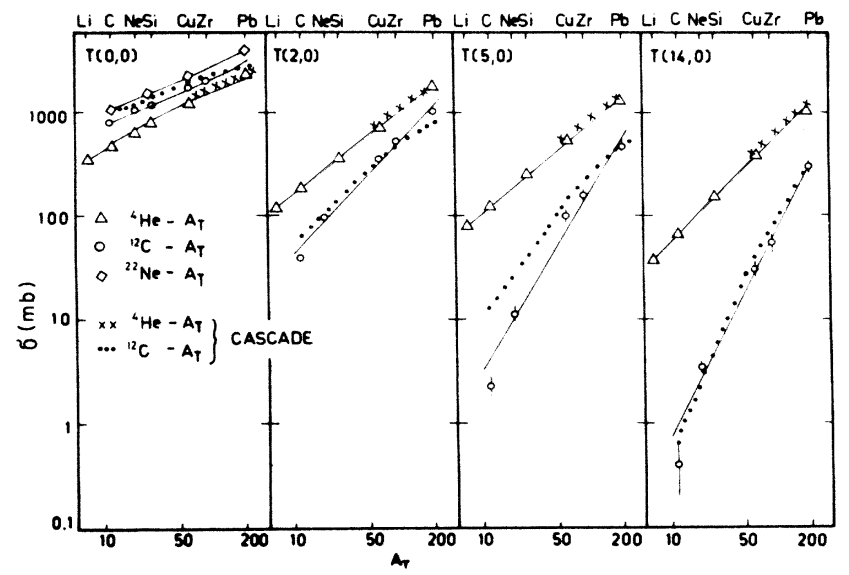

FIG. 3. The dependence of cross section values $\sigma$ on the target mass number $A_{T}$ for various trigger modes. Solid lines show $\sigma^{\text {inel(cent) }}$ dependence on $A_{T}$ derived from formulas given in the text. Dots and crosses show the dependence predicted by the Dubna intranuclear cascade model (DICM).

sections ${ }^{9}$ and only our data on the cross sections for central collisions. The best fit values of the parameters are presented in Table I.

\section{PION MULTIPLICITY}

The shapes of multiplicity distributions for pions produced in nucleus-nucleus collisions are expected to change with the impact parameter of the collision $b$. Figure 4 presents topological cross sections for pions produced in ${ }^{12} \mathrm{C}-\mathrm{Cu}$ interactions for four trigger modes: $T(0,0)$, $T(2,0), T(3,3)$, and $T(14,3)$. The left-hand side of $d \sigma / d n_{\text {- distributions strongly depends on the trigger }}$ mode and consequently on $\langle b\rangle$, whereas the high $n_{-}$part of the multiplicity spectrum is practically trigger independent for three of the trigger modes used: $T(0,0), T(2,0)$, and $T(3,3)$. This observation is consistent with the known fact that high multiplicity events cannot be produced in peripheral collisions. It is seen in the case of the $T(14,3)$ triggering mode that the high multiplicity $\left(n_{-}-{ }^{\prime} 10\right)$ part of the distribution is depleted. This effect is not surprising since the high $\theta_{\text {ch }}$ trigger mode rejects not only peripheral events but also a significant fraction of central ones.

The dependence of $\eta_{n_{-}}=D_{n_{-}}^{2} /\left\langle n_{-}\right\rangle\left(\eta_{n_{-}}\right.$may be used as a measure of the "relative width" of the distribution)

TABLE I. Fitted values of parameters used in formulas describing the dependence of inelastic and central cross sections on $A_{P}$ and $A_{T} . r_{0}$ and $b_{0}$ values are given in $\mathrm{fm}$ and $\mathrm{C}$ values are in $\mathrm{fm}^{2}$.

\begin{tabular}{lcccccc}
\hline \hline & & ${ }^{4} \mathrm{He}$ & & & ${ }^{12} \mathrm{C}$ & \\
& $r_{0}$ & $b_{0}$ & $\chi^{2} / \mathrm{NDF}$ & $r_{0}$ & $b_{0}$ & $\chi^{2} / \mathrm{NDF}$ \\
\hline$T(0,0)$ & 1.4 & 1.03 & 3 & 1.4 & 1.03 & 3 \\
& $\mathrm{C}$ & $\alpha$ & $\chi^{2} / \mathrm{NDF}$ & $\mathrm{C}$ & $\alpha$ & $\chi^{2} / \mathrm{NDF}$ \\
$T(2,0)$ & 2.5 & 0.81 & 0.15 & 0.34 & 1.1 & 2.9 \\
$T(5,0)$ & 1.4 & 0.87 & 0.40 & 0.006 & 1.71 & 6.5 \\
$T(14,0)$ & 0.5 & 1.03 & 0.40 & 0.0007 & 1.99 & 2.4 \\
\hline \hline
\end{tabular}




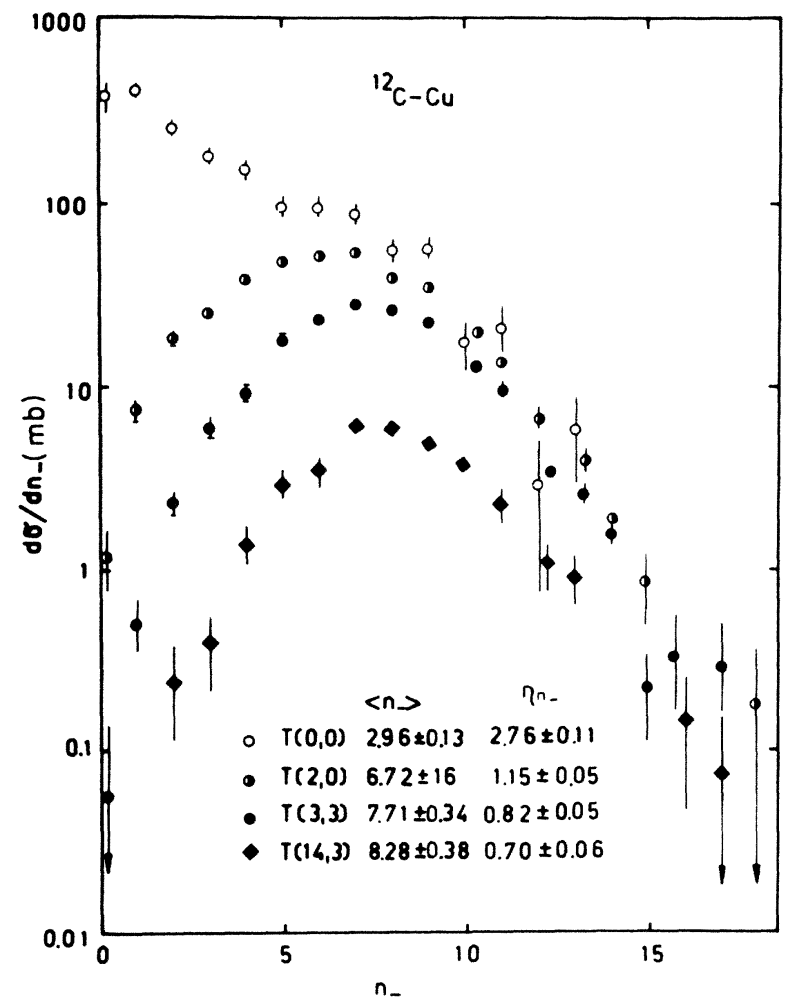

FIG. 4. Topological cross section values, $d \sigma / d n_{-}$, for ${ }^{12} \mathrm{C}-\mathrm{Cu}$ collisions for various trigger modes.

on the collision centrality is shown in Fig. 5. It is seen that the parameter $\eta_{n_{-}}$changes significantly with $p_{T}^{\text {ch }}$ for $p_{T}^{\text {ch }} \approx 250 \mathrm{MeV} / c$. The observation is consistent with the assumption that the transverse momenta of projectile fragments are determined mainly by the Fermi motion of nucleons in the incident nucleus. The dependence of $\eta_{n}$ on the trigger mode for $p_{T}^{\text {ch }} \widetilde{>} p_{F}$ is much less pronounced because the main factor determining the multiplicity, namely the number of interacting nucleons in the incident nucleus, is approximately fixed already for $p_{T}^{\text {ch }} \cong p_{F}$. The influence of $\theta_{n}$ (for $\theta_{\mathrm{ch}} \neq 0$ ) on the $\eta_{n_{-}}$parameter is weak. It is expected that for fixed target nucleus $\langle b\rangle$ decreases with increasing projectile mass number $A_{P}$ provided that all protons of the projectile nucleus have interacted. Therefore the fraction of interacting neutrons increases and the fluctuation of this fraction decreases with increasing $A_{P}$. Consequently it is expected that the influence of $\theta_{n}$ value on multiplicity spectra should be more important for very light projectiles than for heavier ones.

Figure 6 presents $\left\langle n_{-}\right\rangle / A_{P}$ plotted against $A_{T}$ for four groups of trigger modes used in this experiment: (a) $T(0,0)$; (b) $T(2,0), T(2,2)$; (c) $T(5,0), T(5,2), T(5,3)$; and (d) $T(14,0), T(14,2), T(14,3)$. It is seen that the ratio $\left\langle n_{-}\right\rangle / A_{P}$ increases with $A_{T}$ for all trigger modes. In the case of inelastic collisions the data for two different incident nuclei are shifted with respect to each other. The central collision data for various $A_{P}$ values follow the

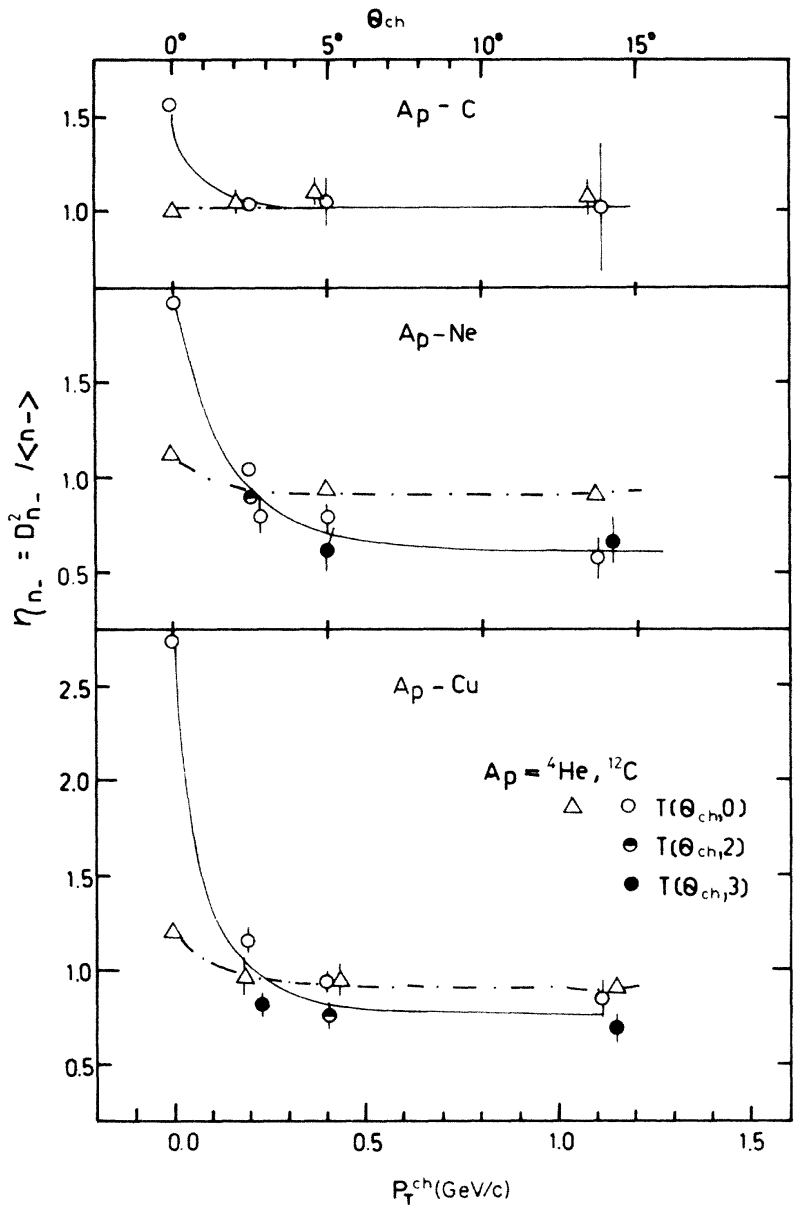

FIG. 5. The dependence of $\eta_{n_{-}}=D_{n_{-}}^{2} /\left\langle n_{-}\right\rangle$on $p_{T}^{\text {ch }}$ (for definition see Fig. 2) for various veto angles for neutral fragments $\theta_{n}$. The solid and dashed-dotted lines are drawn to guide the eye only.

same dependence on $A_{T}$, except of the $\left\langle n_{-}\right\rangle / A_{P}$ values for ${ }^{4} \mathrm{He}-\mathrm{Cu}$ and ${ }^{4} \mathrm{He}-\mathrm{Pb}$ collisions, which are significantly lower than those for ${ }^{12} \mathrm{C}$ and ${ }^{16} \mathrm{O}$ projectiles. This effect can be interpreted as another manifestation of an $A_{P}$ dependence of the fraction of interacting neutrons for $\theta_{\mathrm{ch}} \neq 0^{\circ}$ and $\theta_{n}=0^{\circ}$.

The $\eta_{n_{-}}$values are plotted against $A_{T}$ in Fig. 7. The dependence of the $\eta_{n_{-}}$parameter on $A_{T}$ is much stronger for inelastic interactions than for central ones. The observation can be understood in terms of the following consideration. The width of the multiplicity distribution is determined mainly by fluctuations of the number of nucleons involved in the interaction; in the case of central collisions the fluctuations are suppressed by the triggering conditions and, consequently, $\eta_{n_{-}}$is expected to be much lower and approximately independent of $A_{P}$ and $A_{T}$ values.

The asymmetry of the multiplicity distribution $\left(\gamma_{n_{-}}^{1}\right)$ decreases with $A_{T}$ and collision centrality (see Fig. 8). The values of $\gamma_{n_{-}}^{1}$ obtained for central ${ }^{4} \mathrm{He}-A_{T}$ collisions 


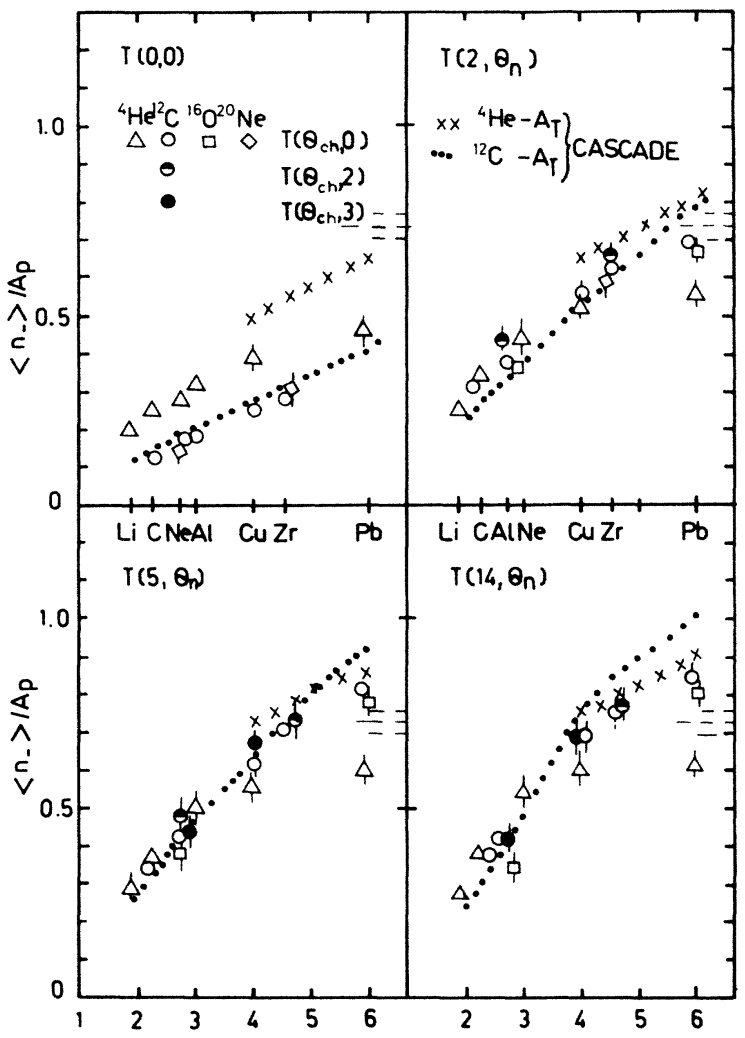

FIG. 6. The dependence of $\left\langle n_{-}\right\rangle / A_{P}$ values on the target mass number $A_{T}$ for four groups of trigger modes $T$. Dots and crosses show the dependence derived from DICM. Dashed lines show limiting $\left\langle n_{-}\right\rangle / A_{P}$ value with its uncertainty corridor obtained within the no-cascading model.

are higher than those obtained for collisions of heavier projectiles $\left({ }^{12} \mathrm{C},{ }^{16} \mathrm{O}\right.$, and $\left.{ }^{20} \mathrm{Ne}\right)$. For the central collisions on heavy targets the asymmetry is close to zero.

\section{KINEMATICAL CHARACTERISTICS OF PIONS}

One can expect that kinematical characteristics of pions depend on the thicknesses of the colliding nuclear layers. It is, therefore, desirable to study the dependence of pion kinematical characteristics on the impact parameter and on mass numbers of colliding nuclei. Since the pion multiplicity $n_{-}$is correlated with the impact parameter $b$ (see Sec. VII) the study of the $b$ dependence of a given variable (like $y, p_{T}$, etc.) can be qualitatively replaced by the study of the dependence of the same variable on $n_{-}$.

Figures 9-14 present the $n_{-} / A_{P}$ dependence of $\langle y\rangle$, $D_{y}, \gamma_{y}^{1},\left\langle p_{T}\right\rangle, D_{p_{T}}$, and $\gamma_{p_{T}}^{1}$, respectively, for three groups of target nuclei. The values derived from experimental $\mathrm{N}-\mathrm{N}$ data ${ }^{19}$ are shown in the figures as dashed lines (the values are averaged over all $n_{-}$values). It is seen that for fixed $A_{T}$ and different values of $A_{P}$ and $n_{-}$, and for various trigger modes, the $y$ and $p_{T}$ data scale in the variable $n_{-} / A_{P}$.

In the case of symmetric (or approximately symmetric, $A_{P} \approx A_{T}$ ) pairs of nuclei the average rapidity $\langle y\rangle$ is independent of $n_{-} / A_{T}$ and it is equal to the rapidity of the

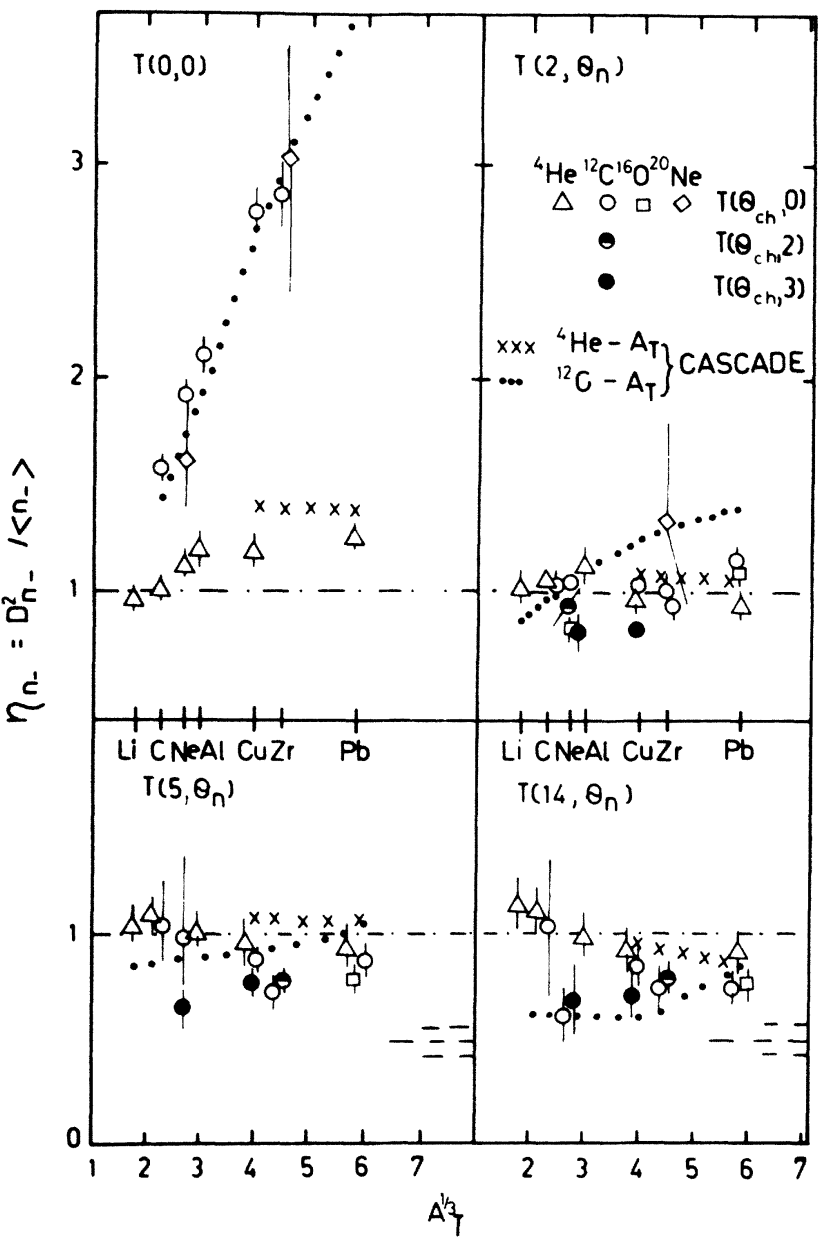

FIG. 7. The dependence of $\eta_{n}$ on $A_{T}$ for four groups of trigger modes. Dots and crosses show the dependence derived from DICM. Dashed-dotted lines show $\eta_{n}=1$ (Poisson distribution). Dashed lines show the limiting value of $\eta_{n}$ with its uncertainty corridor obtained within the no-cascading model.

N-N c.m. system, $y^{\mathrm{NN}}$ [Fig. 9(a)]. This observation is obviously expected from the symmetry of the colliding system. Note that our definition of a central collision (see Sec. II, triggering criteria) introduces some asymmetry, nevertheless our estimation of this effect and bias sources showed that they are negligible. ${ }^{15}$

In the case of asymmetric systems of colliding nuclei $\left(A_{P}<A_{T}\right)$ it is seen [Figs. 9(b) and (c)] that (i) $\langle y\rangle$ decreases from $y^{\mathrm{NN}}$ (for the lowest $n_{-} / A_{P}$ values) to 0.5 $y^{\mathrm{NN}}$ (for highest $n_{-} / A_{P}$ values and for the heaviest target, $\mathrm{Pb})$ and (ii) $\langle y\rangle$ decreases with increasing $A_{T}$ for fixed $n_{-} / A_{P}$ values. The dispersion of the rapidity distribution $D_{y}$ (Fig. 10) for low $n_{-} / A_{P}$ values is consistent with that for N-N collisions and it decreases with increasing $n_{-} / A_{P}$.

The skewness parameter $\gamma_{y}^{1}$ is close to zero for sym- 


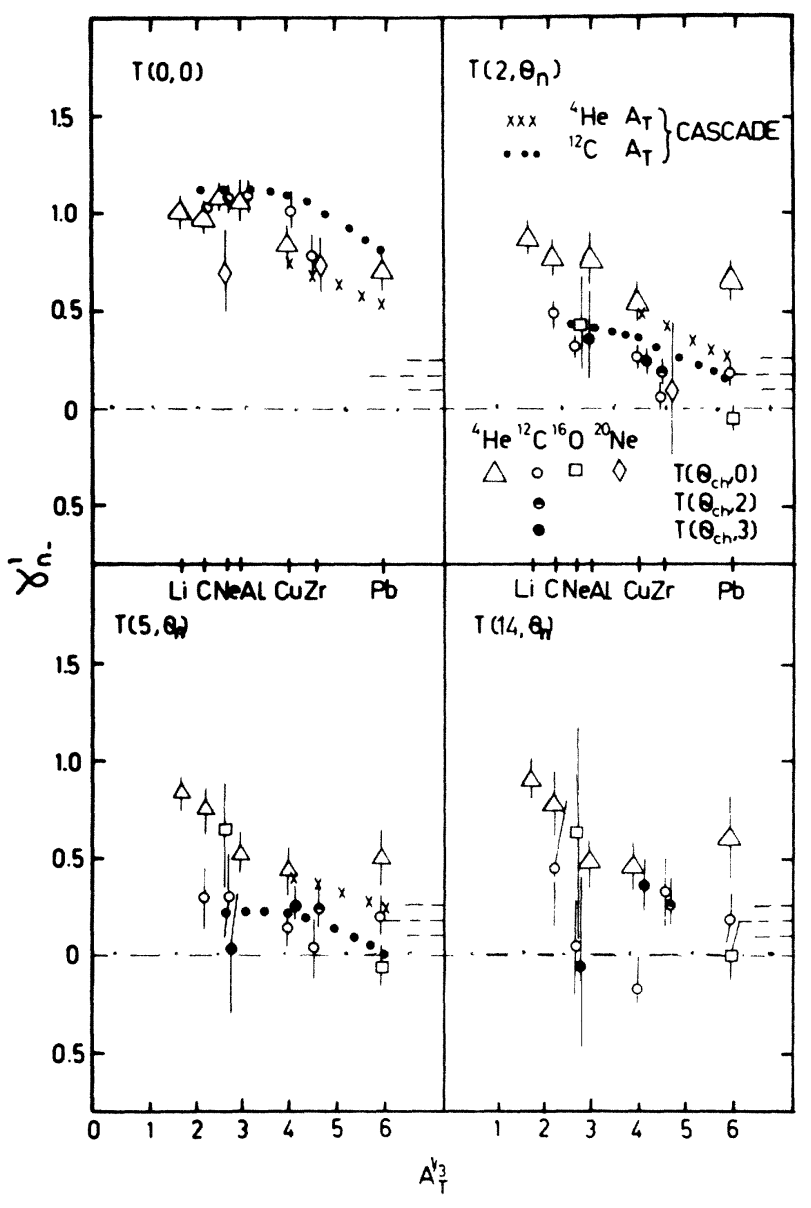

FIG. 8. The dependence of $\gamma_{n_{-}}^{1}$ on $A_{T}$ for four groups of trigger modes. Dots and crosses show the dependence derived from DICM. Dashed-dotted lines show $\gamma_{n_{-}}^{1}=0$ (symmetric distribution). Dashed lines show the limiting value of $\gamma_{n_{-}}^{1}$ with its uncertainty corridor obtained within the no-cascading model.

metric systems [Fig. 11(a)], which has been, of course, expected, whereas $\gamma_{y}^{1}$ data lie systematically over the zero line for asymmetric colliding nuclei. The effect is most pronounced for the heaviest target, $\mathrm{Pb}$, and for high $n_{-} / A_{P}$ values [Figs. $11(\mathrm{~b})$ and (c)].

Average values of transverse momentum $\left\langle p_{T}\right\rangle$ for low $n_{-} / A_{P}$ values are close to those observed in $\mathrm{N}-\mathrm{N}$ collisions and they decrease (i) with $n_{-} / A_{P}$ for fixed $A_{T}$ and (ii) with $A_{T}$ for fixed $n_{-} / A_{P}$ values (Fig. 12). Figure 13 does not show any significant dependence of $D_{p_{T}}$ on $n_{-} / A_{P}$ or $A_{T}$, if slightly lower $D_{p_{T}}$ values for high $n_{-} / A_{P}$ in the case of the $\mathrm{Pb}$ target are disregarded.

The $\gamma_{p_{T}}^{1}$ parameter values (Fig. 14) do not exhibit any significant dependence on $n_{-} / A_{P}$ or $A_{T} \cdot \gamma_{p_{T}}^{1}$ values are systematically higher than those observed in N-N collisions.

In Fig. 15 average transverse momentum values are plotted against $\langle y\rangle$ for samples of events characterized by fixed $A_{P}, A_{T}$, and the trigger mode for several intervals

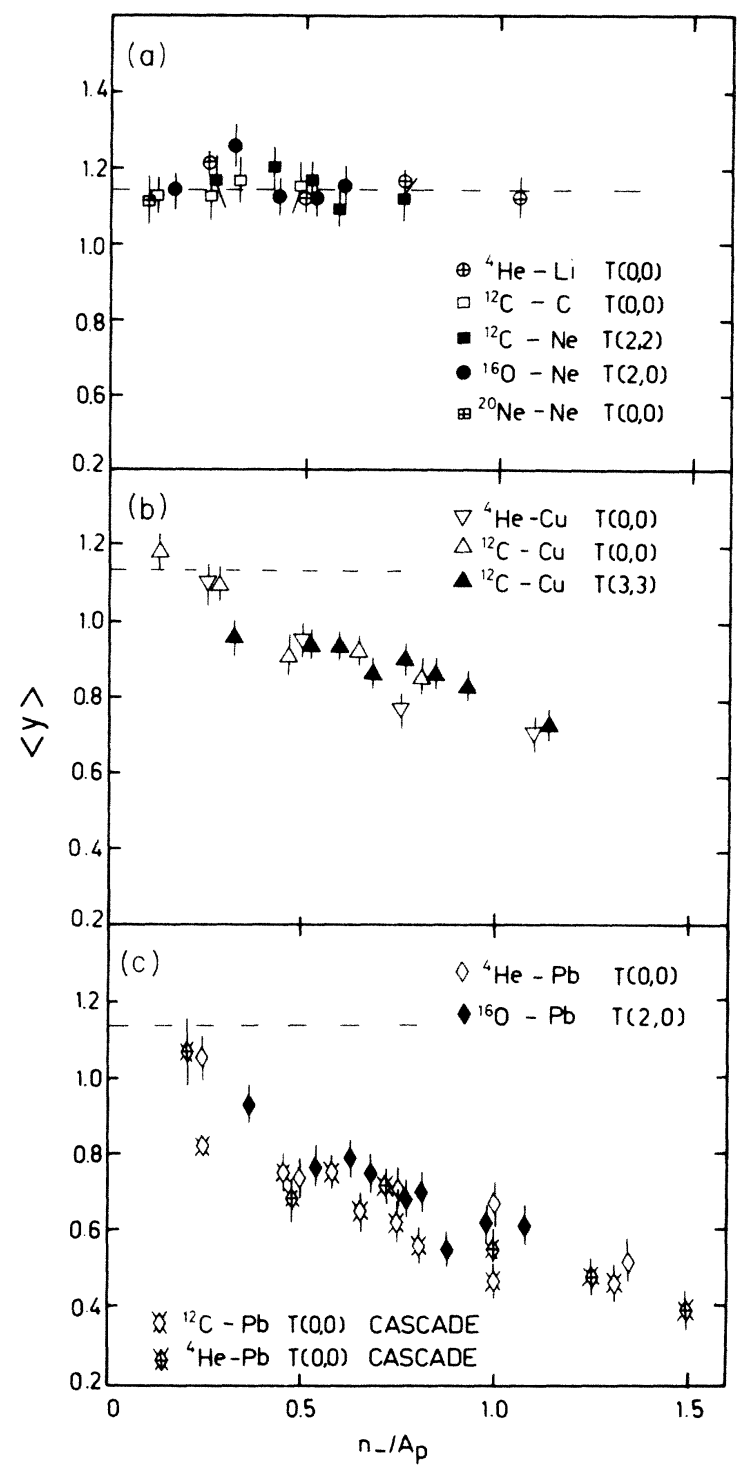

FIG. 9. The dependence of the average rapidity $\langle y\rangle$ on the $n_{-} / A_{P}$ variable for various trigger modes: (a) light target nuclei; (b) intermediate target nucleus, $\mathrm{Cu}$; (c) heavy target nucleus, $\mathrm{Pb}$. Results of calculations within the DICM are plotted for the $\mathrm{Pb}$ target only. Dashed lines show the $\langle y\rangle$ value for $\mathrm{N}-\mathrm{N}$ collisions at $4.5 \mathrm{GeV} / c$.

of pion multiplicity $n_{-}$. All data seem to follow the same roughly linear dependence of $\left\langle p_{T}\right\rangle$ on $\langle y\rangle$. The same scaling in $\langle y\rangle$ was observed for other parameters of $y$ and $p_{T}$ distributions (not shown here).

The fact that $y$ distributions are almost symmetric (Fig. 11) might suggest that pions produced in nucleus-nucleus collisions are emitted symmetrically with respect to the $y=\langle y\rangle$ axis. A somewhat more detailed analysis of the $p_{T}$ vs $y$ diagram for pions from asymmetric systems (not presented here) shows, however, that such an interpretation is not correct. The average rapidity depends on $p_{T}$ as is shown in Fig. 16 for collisions with the $\mathrm{Pb}$ target.

In the case of collisions of nuclei of approximately 


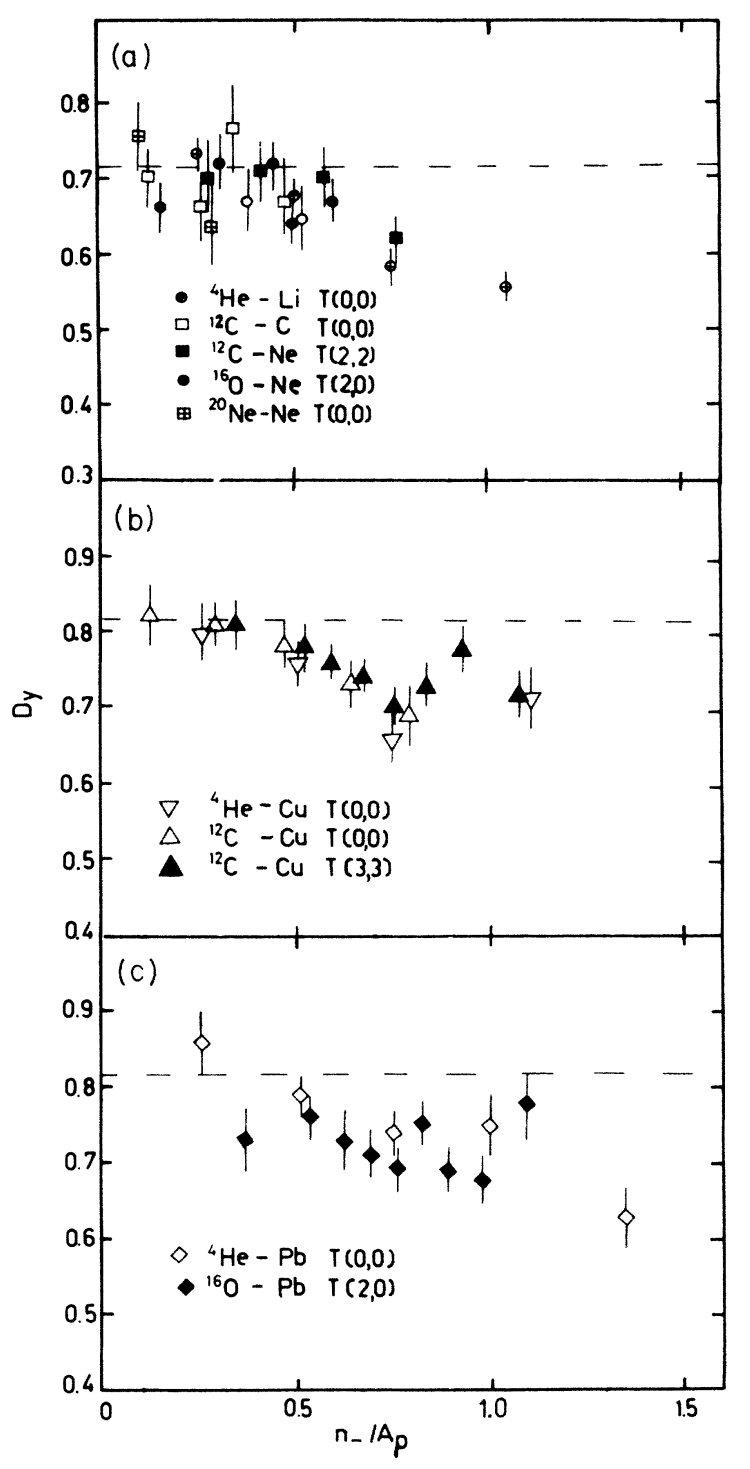

FIG. 10. The dependence of the dispersion values of the rapidity distribution $D_{y}$ on the $n_{-} / A_{P}$ variables for various trigger modes: (a) light target nuclei; (b) intermediate target nucleus, $\mathrm{Cu}$; (c) heavy target nucleus, $\mathrm{Pb}$. Dashed lines show the $D_{y}$ value for $\mathrm{N}-\mathrm{N}$ collisions at $4.5 \mathrm{GeV} / c$.

equal masses the $p_{T^{-}-y}$ plots are symmetric with respect to the $y=y_{\text {c.m. }}=y^{\mathrm{NN}}$ line. The analysis of the dependence of the dispersion of the $\cos \theta^{*}$ distribution on $n_{-} / A_{P}$ and $E^{*}\left(\theta^{*}\right.$ and $E^{*}$ are emission angle and kinetic energy in the N-N c.m. system, respectively) is expected to yield further insight into the pion emission mechanism. Figure 17 shows that $D_{\cos \theta^{*}}$ decreases with $n_{-} / A_{P}$ approaching the $D_{\cos \theta^{*}}$ value corresponding to an isotropic distribution (0.57) for the inelastic ${ }^{4} \mathrm{He}-\mathrm{Li}$ collisions for the highest $n_{-} / A_{P}$ values. The decrease is not seen for central ${ }^{12} \mathrm{C}-\mathrm{Ne}$ and ${ }^{16} \mathrm{O}-\mathrm{Ne}$ and inelastic ${ }^{12} \mathrm{C}-\mathrm{C}$ collisions.

Figure 18 shows a monotonic increase of $D_{\cos \theta^{*}}$ with $E^{*} / E_{\max }^{*}$ for all groups of events of colliding nuclei with close mass numbers, including ${ }^{4} \mathrm{He}-\mathrm{Li}$ inelastic collisions.

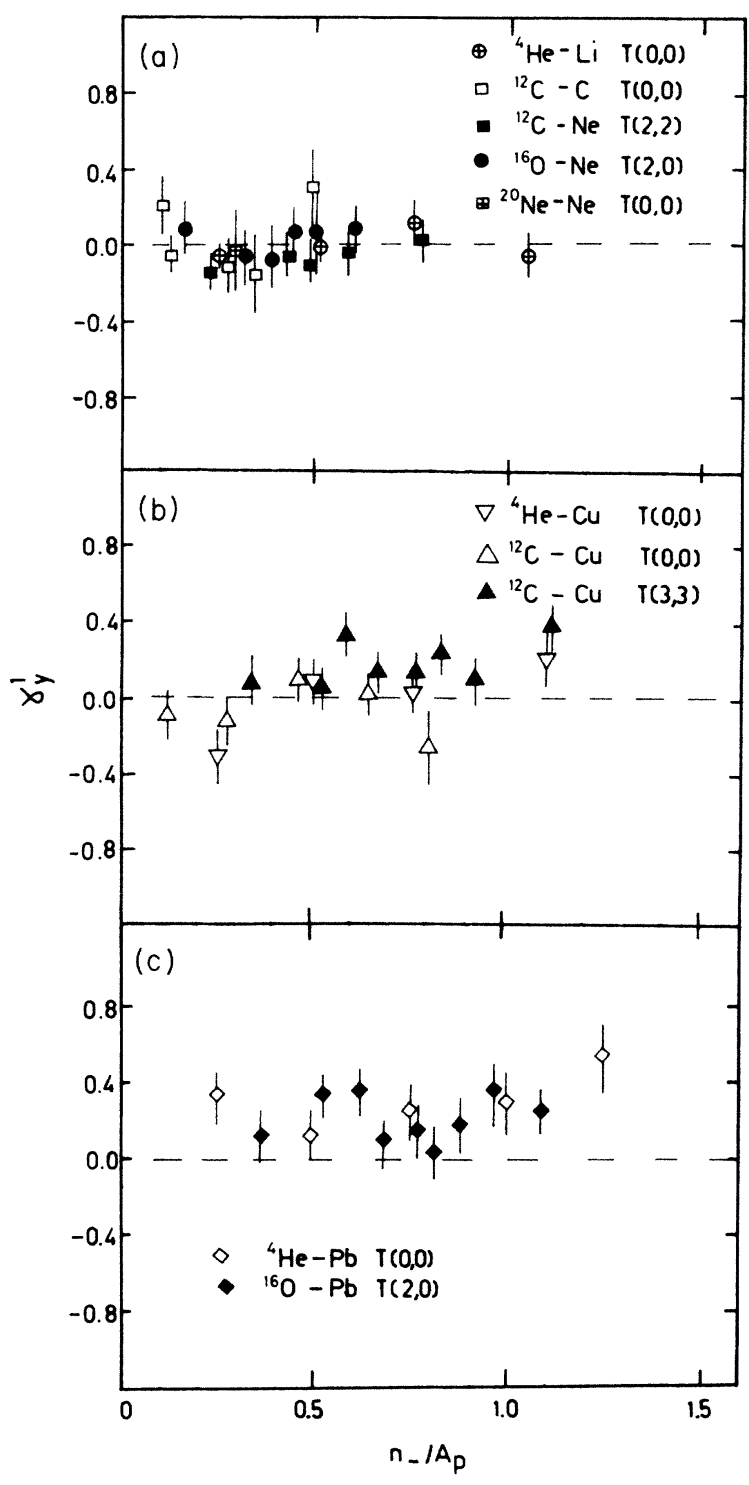

FIG. 11. The dependence of the skewness parameter of the rapidity distribution, $\gamma_{y}^{1}$, on the $n_{-} / A_{P}$ variable for various trigger modes: (a) light target nuclei; (b) intermediate target nucleus, $\mathrm{Cu}$; (c) heavy target nucleus, $\mathrm{Pb}$. Dashed lines show the $\gamma_{y}^{1}\left(\gamma_{y}^{1}=0\right)$ value for $\mathrm{N}-\mathrm{N}$ collisions.

A similar analysis has been performed for Ar- $\mathrm{KCl}$ collisions at $2.6 \mathrm{GeV} / c$ nucleons. ${ }^{20(a)}$ The origin of the different behavior of the pion angular distributions as a function of the pion energy [the broadening of distributions in our experiment and the narrowing of distributions in the Lawrence Berkeley Laboratory (LBL) experiment for energetic pions] seems to be connected with lower primary energy and higher masses of projectiles in the LBL experiment than in ours.

\section{CORRELATIONS OF PION EMISSION WITH EMISSION OF OTHER PARTICLES}

The data obtained in this experiment concern, beside the pion emission, the production of strange particles, in 


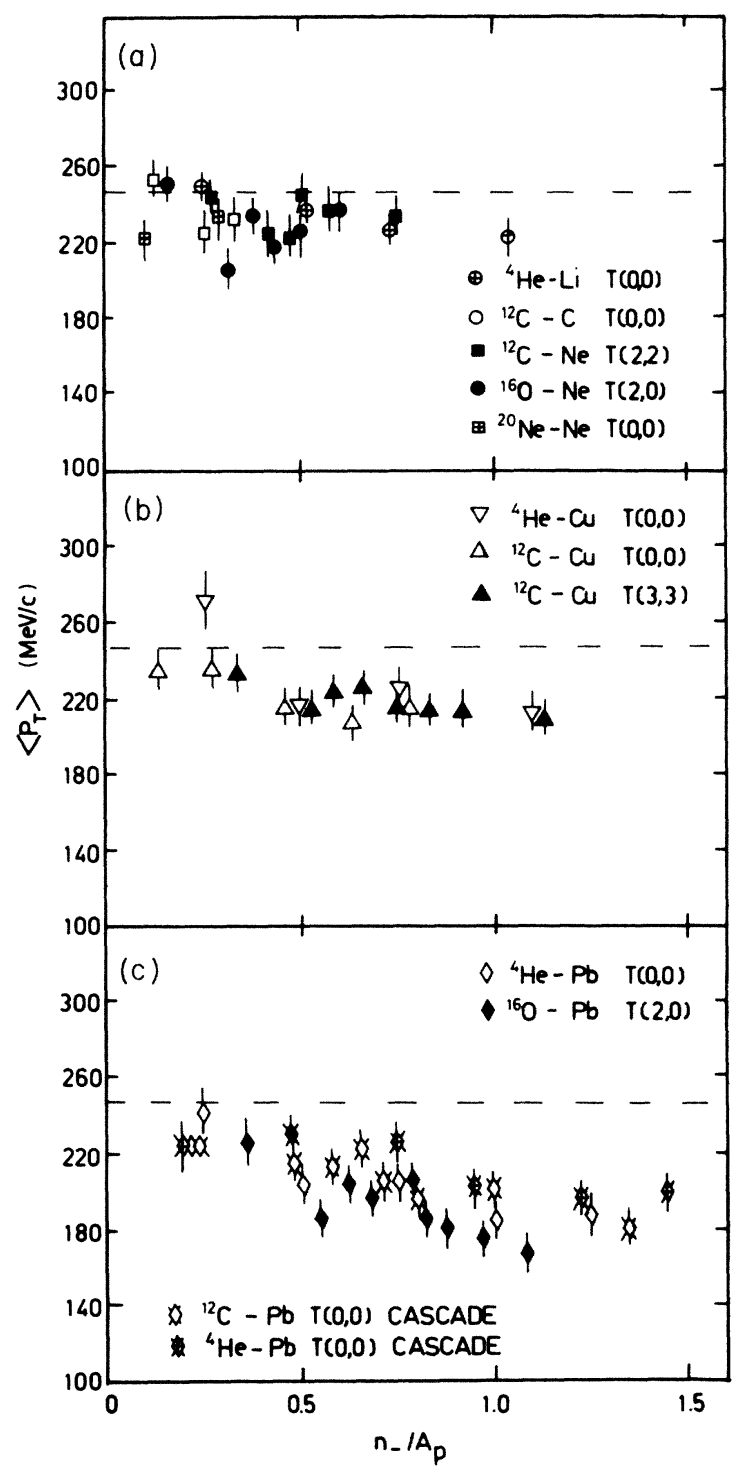

FIG. 12. The dependence of the average transverse momentum, $\left\langle p_{T}\right\rangle$, on the $n_{-} / A_{P}$ variable for various trigger modes: (a) light target nuclei; (b) intermediate target nucleus, $\mathrm{Cu}$; (c) heavy target nucleus, $\mathrm{Pb}$. Results of calculations within the $\mathrm{DICM}$ are plotted for the $\mathrm{Pb}$ target only. Dashed lines show the $\left\langle p_{T}\right\rangle$ value for $\mathrm{N}-\mathrm{N}$ collisions at $4.5 \mathrm{GeV} / c$.

particular $\Lambda$ hyperons, and the emission of positive secondaries with $p / Z>300 \mathrm{MeV} / c$ (mainly protons) into the backward hemisphere in the laboratory system. These data are discussed in detail in Refs. 12-14 and 16. The data on $\Lambda$ and "backward" protons are used here for a search of possible correlations with the pion production.

No cuts or corrections due to the efficiency of $\Lambda$ registration were used in this analysis in order to increase the number of events with $\Lambda$ production. It has been checked that in the case of the analysis of the multiplicity of pions associated with $\Lambda$ the results obtained with and without the above corrections are the same. When analyzing the kinematical characteristics of the pion no spurious correlations due to using uncorrected samples are expected.

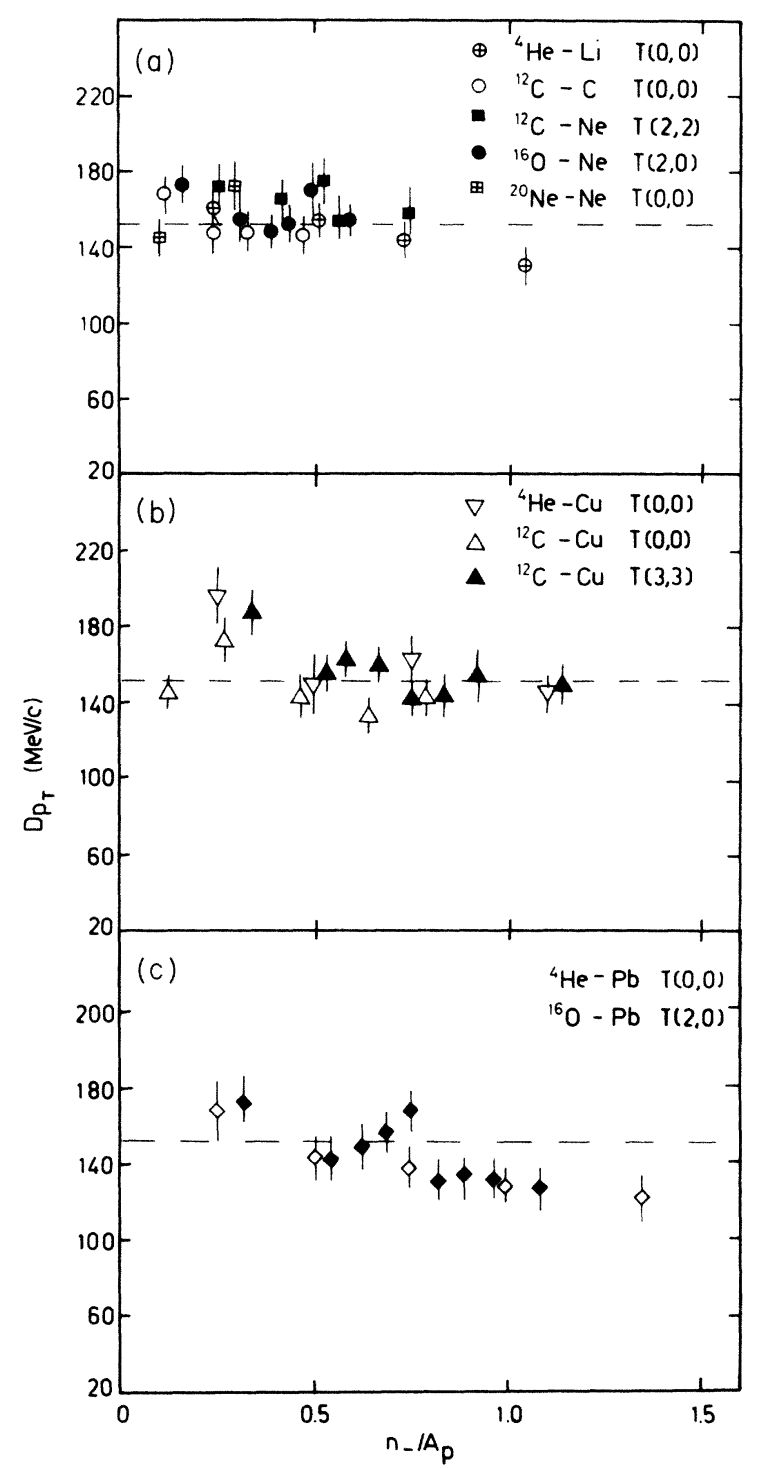

FIG. 13. The dependence of the dispersion values of transverse momentum distributions, $D_{p_{T}}$, on the $n_{-} / A_{P}$ variable for various trigger modes: (a) light target nuclei; (b) intermediate target nucleus, $\mathrm{Cu}$; (c) heavy target nucleus, $\mathrm{Pb}$. Dashed lines show the $D_{p_{T}}$ value for $\mathrm{N}-\mathrm{N}$ collisions at $4.5 \mathrm{GeV} / c$.

The values of the ratio of pion yields for events in which a $\Lambda$ hyperon has been emitted, $\left\langle n_{-}\right\rangle^{\Lambda}$, and for "ordinary" (without $\Lambda$ production) events, $\left\langle n_{-}\right\rangle^{\mathrm{no} \Lambda}$, are shown in Fig. 19(a). It is seen that in the case of inelastic ${ }^{4} \mathrm{He}-\mathrm{Li}$ collisions the average pion multiplicity is higher in " $\Lambda$ events" than in ordinary events, whereas in the case of central collisions $\left\langle n_{-}\right\rangle^{\Lambda}$ is close to $\left\langle n_{-}\right\rangle^{\text {no } \Lambda}$ as has been observed earlier in $\mathrm{Ar}-\mathrm{KCl}$ collisions at 2.6 $\mathrm{GeV} / \mathrm{c} \mathrm{nucleon}^{20(\mathrm{~b})}$ This observation is not surprising since it can be expected that $\Lambda$ 's are more abundantly produced in central collisions (characterized by high multiplicity) than in inelastic ones.

No significant correlation of the pion multiplicities with the $\Lambda$ hyperon momentum was found. Pion and $\Lambda$ 


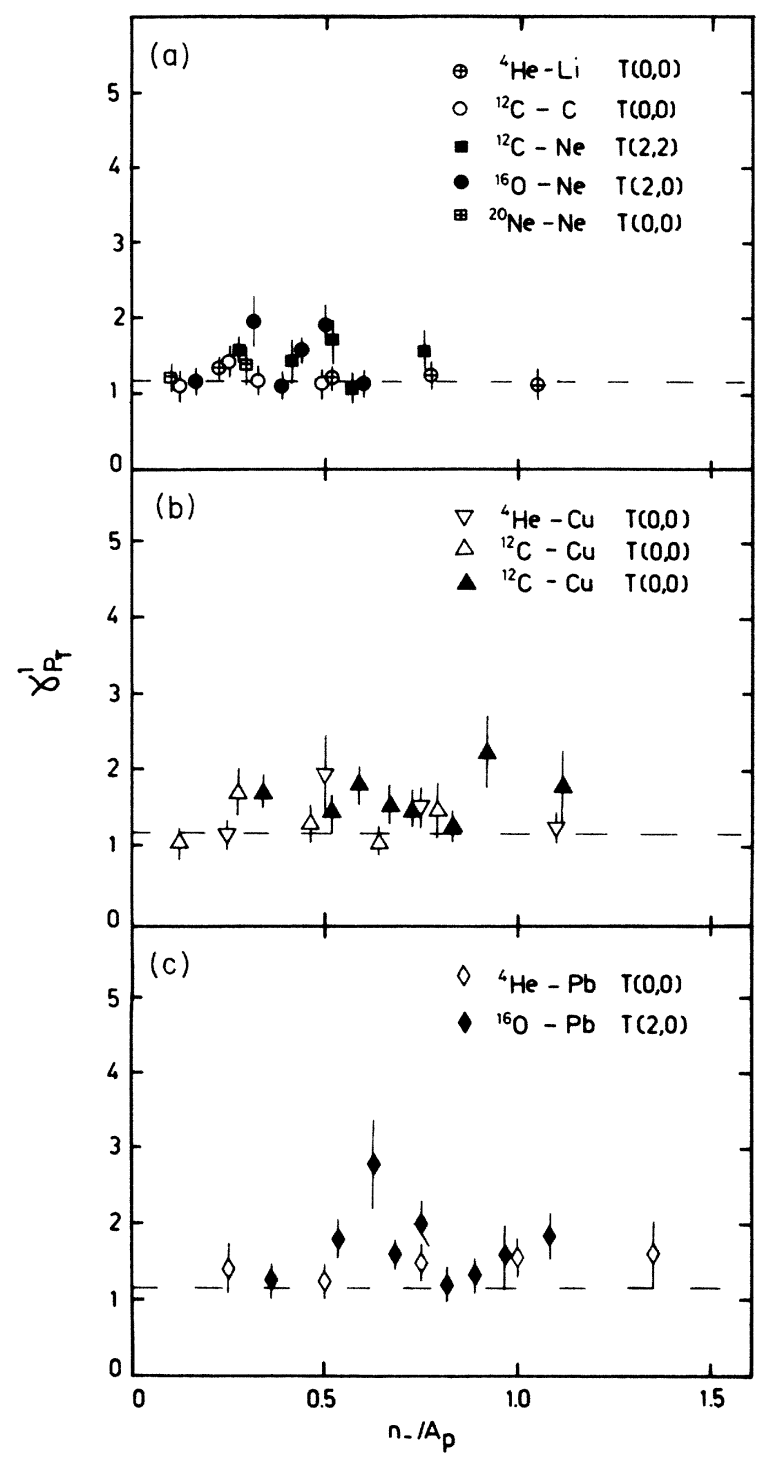

FIG. 14. The dependence of the skewness values of transverse momentum distributions, $\gamma_{p_{T}}^{1}$, on the $n_{-} / A_{P}$ variable for various trigger modes: (a) light target nuclei; (b) intermediate target nucleus, $\mathrm{Cu}$; (c) heavy target nucleus, $\mathrm{Pb}$. Dashed lines show the $\gamma_{p_{T}}^{1}$ value for $\mathrm{N}-\mathrm{N}$ collisions at $4.5 \mathrm{GeV} / c$.

emission angles seem also to be uncorrelated.

A correlation of pion kinematical characteristics with $\Lambda$ 's momentum in the N-N c.m. system was found in central ${ }^{12} \mathrm{C}-\mathrm{C},{ }^{12} \mathrm{C}-\mathrm{Ne}$, and ${ }^{16} \mathrm{O}-\mathrm{Ne}$ collisions. Two subsamples of events were considered:

(a) events with a $\Lambda$ produced within the $\mathrm{N}-\mathrm{N}$ kinematical limits for $N-N$ collision (" $\Lambda$ in" events); and

(b) events with a $\Lambda$ produced beyond the $\mathrm{N}-\mathrm{N}$ kinematical limits (" $\Lambda$ out" events).

We find that $d n_{-} / d \cos \theta^{*}$ (Fig. 20) and $d E^{*} / d \cos \theta^{*}$ spectra $\left(\theta^{*}\right.$ and $E^{*}$ are pion emission angle and kinetic energy in the $\mathrm{N}-\mathrm{N}$ system, respectively) are flat for $\Lambda$ out events in contrast to the spectra observed for ordinary

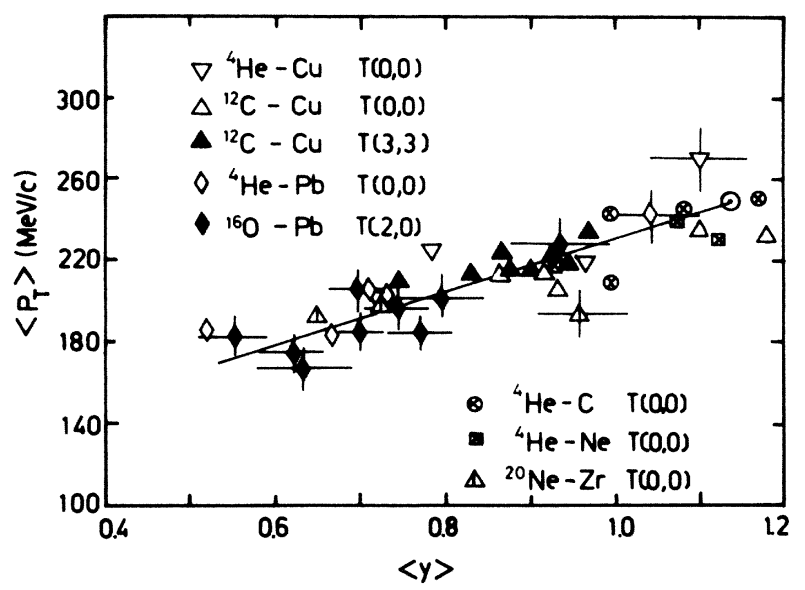

FIG. 15. $\left\langle p_{T}\right\rangle-\langle y\rangle$ dependence for various trigger modes, various pairs of colliding nuclei, and pion multiplicities. The solid line is the linear fit to experimental points. $\odot$ shows the $\mathrm{N}-\mathrm{N}$ point for our energy.

central events and $\Lambda$ in events. ${ }^{21}$ The average transverse momentum and its dispersion are higher for $\Lambda$ out pions than for pions from ordinary central events $\left[\left\langle p_{T}\right\rangle=(279 \pm 21) \mathrm{MeV} / c, D_{p_{T}}=(191 \pm 22) \mathrm{MeV} / c\right.$, and $\left\langle p_{T}\right\rangle=(234 \pm 6) \mathrm{MeV} / c, D_{p_{T}}=(164 \pm 8) \mathrm{MeV} / c$, respectively].

Characteristics of pions from " $\Lambda$ in" events and from " $\Lambda$ out" events for intermediate target nuclei do not reveal any significant difference when compared with corresponding distributions of pions produced in ordinary central events (see Fig. 20). It should be noted, however, that the origin of the $\Lambda$ hyperons produced beyond the $\mathrm{N}-\mathrm{N}$ kinematical limit in light $A_{P} \approx A_{T}$ collisions and in ${ }^{12} \mathrm{C}-\mathrm{Cu}$ and ${ }^{12} \mathrm{C}-\mathrm{Zr}$ collisions seems to be different. A large fraction of the " $\Lambda$ out" hyperons in the latter cases are produced in the target fragmentation region, ${ }^{13}$ whereas in the former case the " $\Lambda$ out" hyperons are produced mostly in the central region of rapidity.

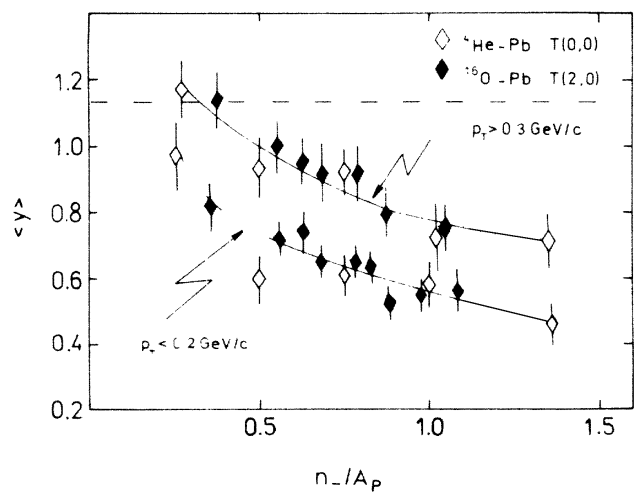

FIG. 16. The dependence of $\langle y\rangle$ for the $\mathrm{Pb}$ target on the $n_{-} / A_{P}$ variable for two intervals of $p_{T}$. Solid lines are drawn to guide the eye only. Dashed line shows the $\langle y\rangle$ value for $\mathrm{N}-\mathrm{N}$ collisions at $4.5 \mathrm{GeV} / c$. 


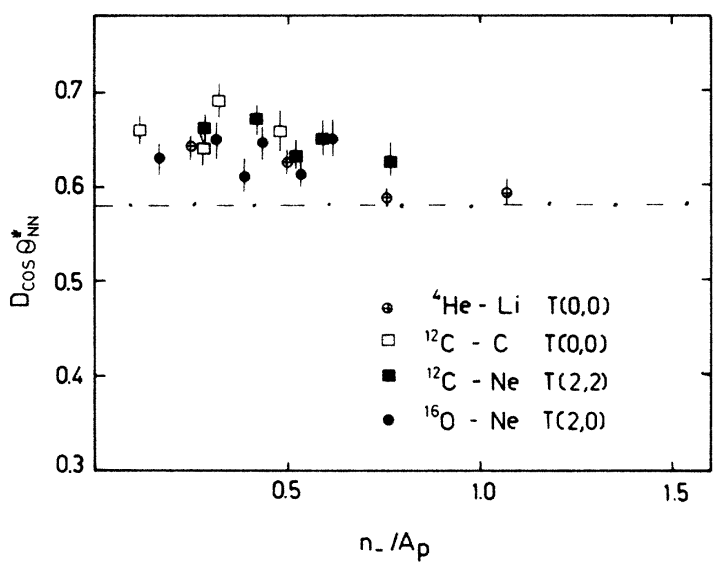

FIG. 17. The dependence of the dispersion of $\cos \theta^{*}$ distributions $\left(\theta^{*}-\pi^{-}\right.$emission angle in the $\mathrm{N}-\mathrm{N}$ c.m. system) on the $n_{-} / A_{P}$ variable. Dashed-dotted line shows the value corresponding to an isotropical angular distribution.

A similar analysis was carried out for pions produced in events in which a charged positive secondary with $p / Z>300 \mathrm{MeV} / c$ has been emitted (" $p$ " events) into the backward hemisphere in the laboratory system. The average pion multiplicity is higher in the $p$ events than in ordinary (without $p$ emission) inelastic and central collisions [Fig. 19(b)]. The highest values of the $\left\langle n_{-}\right\rangle$"p" $/\left\langle n_{-}\right\rangle^{\text {no }}$ " $"$ " ratio are observed for inelastic collisions with heavy targets. No significant correlations between kinematical features of pions and "backward protons" are observed.

\section{COMPARISON OF EXPERIMENTAL RESULTS WITH PREDICTIONS OF THEORETICAL MODELS}

Several theoretical models concerning nucleus-nucleus collisions at high energy have been proposed. ${ }^{1}$ The models allow to test various assumptions concerning the mechanism of particle production at extreme conditions

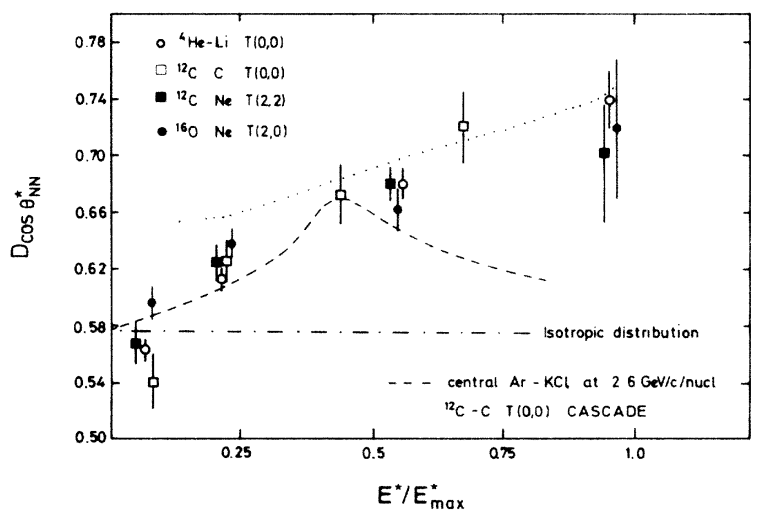

FIG. 18. The dependence of the dispersion of $\cos \theta^{*}$ distributions on the $E^{*} / E_{\max }^{*}$ variable for several trigger modes of nuclear collisions with light target nuclei. $\theta^{*}, E^{*}, E_{\max }^{*}-\pi^{-}$emission angle, kinetic energy, and maximum available kinetic energy in N-N collisions, respectively, in the $\mathrm{N}-\mathrm{N}$ c.m. system.

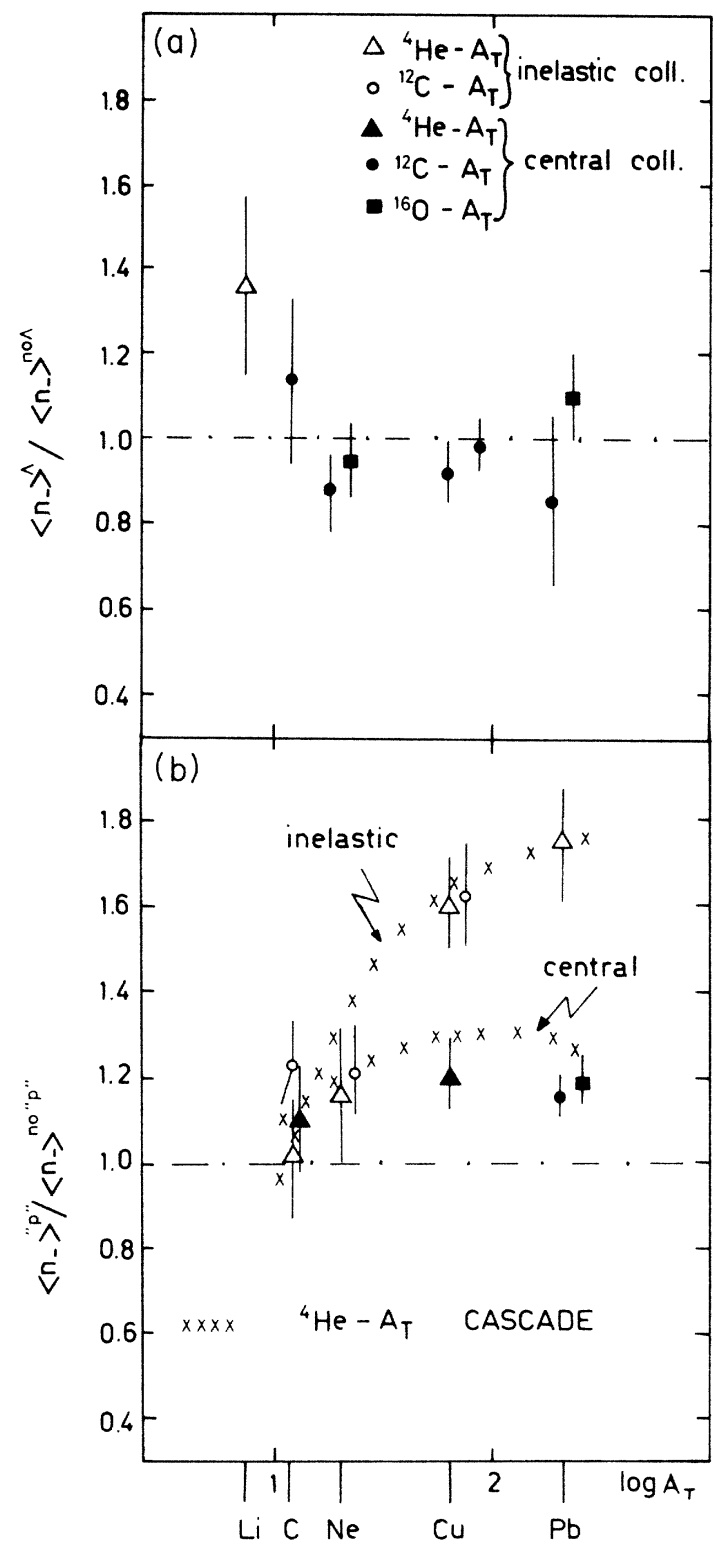

FIG. 19. The ratio of the $\pi$ multiplicity in (a) " $\Lambda$ events," $\left\langle n_{-}\right\rangle^{\Lambda}$, and (b) " $p$ events," $\left\langle n_{-}\right\rangle$" $"$ to that in the "ordinary" collisions plotted against $A_{T}$.

achieved only in nucleus-nucleus collisions. In this section three theoretical approaches are tested: "nocascading" model, internuclear cascade model, and thermodynamic approach.

The first two models are based on the assumption that particles are produced in independent hadron-hadron collisions. Our earlier results ${ }^{8-10}$ obtained from the study of large cross section processes seem to support this assumption.

In the case of the thermodynamic approach one assumes that particle production is determined only by the collision geometry and available phase space. A comparison of the data with predictions of thermodynamic models may yield information about the degree of equili- 


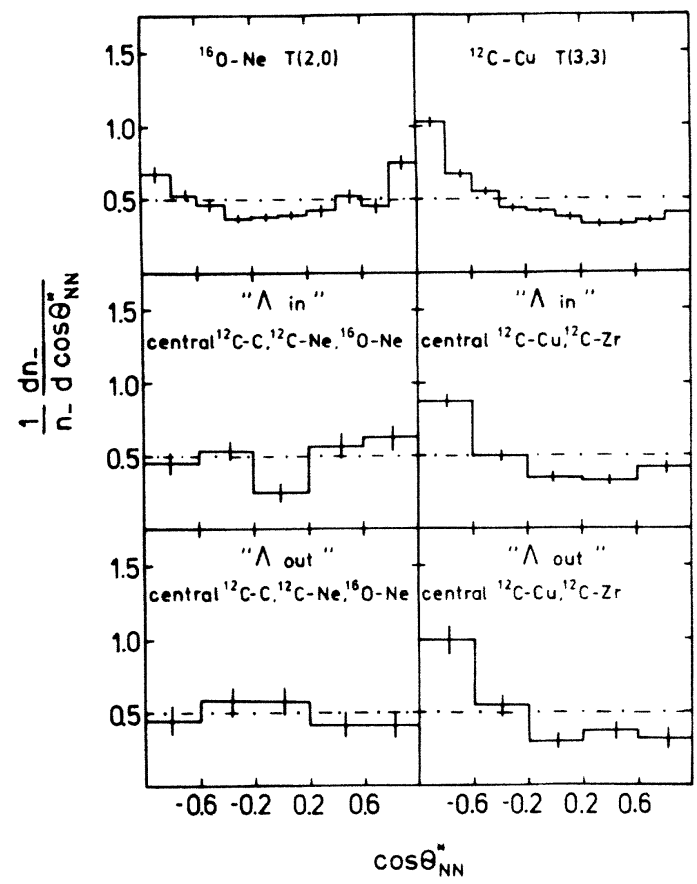

FIG. 20. Angular distributions of pions calculated in the $\mathrm{N}-\mathrm{N}$ c.m. system for several samples of events (for details see the text).

bration of excited nuclear matter. A comparison of our data with some other types of models (e.g., the coherent tube model) can be found in Refs. 8 and 22. Predictions of these models strongly disagree with the data.

\section{A. "No-cascading" model}

"No-cascading" model assumes that each nucleon from projectile and target nuclei can undergo at most one inelastic collision and secondary particles do not interact. Nucleus-nucleus interaction is thus considered as a superposition of independent nucleon-nucleon collisions. This simple picture can be treated as a natural background against which nuclear effects may be looked for. The model predicts the upper limit of the pion multiplicity in an $A_{P}-A_{T}$ collision for the case when all nucleons of the lighter of two colliding nuclei have undergone inelastic interactions. This maximum value of the pion multiplicity is given by the following formula:

$$
\left\langle n_{-}\right\rangle_{\max }=\min \left(A_{P}, A_{T}\right)\left\langle n_{-}\right\rangle^{\mathrm{NN}}
$$

In our experiment $A_{P}$ is smaller than $A_{T}$ and the projectile nuclei $\left({ }^{4} \mathrm{He},{ }^{12} \mathrm{C},{ }^{16} \mathrm{O}\right.$, and $\left.{ }^{20} \mathrm{Ne}\right)$ are isoscalars, therefore

$$
\left\langle n_{-}\right\rangle_{\max }=A_{P}\left\langle n_{-}\right\rangle^{\mathrm{NN}},
$$

where

$$
\begin{aligned}
\left\langle n_{-}\right\rangle^{\mathrm{NN}}= & \frac{1}{2}\left\langle n_{-}\right\rangle^{\mathrm{np}}+\frac{1}{2} \frac{A_{T}-Z_{T}}{A_{T}}\left\langle n_{-}\right\rangle^{\mathrm{nn}} \\
& +\frac{1}{2} \frac{Z_{T}}{A_{T}}\left\langle n_{-}\right\rangle^{\mathrm{pp}} .
\end{aligned}
$$

A similar consideration allows us to calculate the minimum value of the dispersion and the skewness of a multiplicity distribution.

Horizontal dashed lines in Fig. 6 correspond to $\left\langle n_{-}\right\rangle / A_{P}$ values derived from N-N data ${ }^{19}$ by use of the formula given above with the Fermi motion of nucleons taken into account. It is seen that the experimental values approach (but they do not exceed) those derived from the formula, except the $\left\langle n_{-}\right\rangle / A_{P}$ results for the "most central" collisions with the heaviest of our targets, $\mathrm{Pb}$. The observed increase of $\left\langle n_{-}\right\rangle / A_{P}$ with $A_{T}$ for central collisions can be explained due to two processes:

(a) The increase of the average number of interacting protons and/or neutrons with $A_{T}$ for fixed and small $\theta_{\mathrm{ch}}$ and $\theta_{\mathrm{n}}$ values.

(b) The increase of the ratio of inelastically to elastically interacting nucleons with $A_{T}$.

Values of $\eta_{n_{-}}=D_{n_{-}}^{2} /\left\langle n_{-}\right\rangle$and $\gamma_{n_{-}}^{1}$ derived from $\mathrm{N}-\mathrm{N}$ data under the assumption that all $A_{P}$ nucleons of the projectile nucleus have interacted inelastically are presented by dashed lines in Figs. 7 and 8 . It is seen that our experimental $\eta_{n_{-}}$values are higher than the limiting value expected within the no-cascading model, whereas the $\gamma_{n_{-}}^{1}$ values approach the limiting value for central collisions with heavy targets.

The comparison of kinematical characteristics $\left(p_{T}\right.$ and $y$ ) of pions emitted from nucleus-nucleus collisions with the data measured in $\mathrm{N}-\mathrm{N}$ interactions ${ }^{19}$ is presented in Figs. 9-14. The latter data are expected to coincide with predictions of the no-cascading model, if some influence of trivial nuclear effects such as the Fermi motion and different isospin content of interacting nuclei is disregarded. Figures 9-14 show that the values of the analyzed moments of rapidity and transverse momentum distributions are consistent with those observed in $\mathrm{N}-\mathrm{N}$ collisions only for low $n_{-} / A_{P}$ values. The disagreement seen for higher values of the $n_{-} / A_{P}$ variable may be attributed to the nuclear effects, which are essential for high multiplicity collisions (low impact parameter).

Within the no-cascading model the $\Lambda$ production probability increases with the number of inelastically interacting nucleons of the projectile nuclei. Thus, the ratio $\left\langle n_{-}\right\rangle^{\Lambda} /\left\langle n_{-}\right\rangle^{\mathrm{no} \Lambda}$ is expected to be greater than unity and decrease with $A_{T}$ for inelastic collisions. For central collisions, when the number of inelastically interacting nucleons from the projectile is roughly fixed, the ratio should be weakly dependent on $A_{T}$ and close to unity. The experimental data agree with these predictions.

\section{B. Intranuclear cascade model}

The intranuclear cascade model assumes that a nucleus-nucleus interaction consists of a series of subse- 
quent hadron-hadron collisions. The mathematical approach to the solution of this problem is based on the relativistic Boltzmann equation. There are several versions of the cascade model. In this paper the Dubna intranuclear cascade model (DICM) (Refs. 23-25) is used for a comparison with experimental data. Main assumptions and features of the model are presented below. Each of the colliding nuclei is treated as a gas of nucleons moving within a potential well, i.e., nucleons are bound within a nucleus. The distribution of the nucleon density and of the nuclear potential are taken into account as well as correlations between the nucleons within the nucleus in its ground state. It is assumed that the distance between the nucleons is not less than twice the value of the nucleon core radius. ${ }^{23}$ Approximations of the experimental data on elastic and inelastic hadron-hadron collisions are used and, consequently, final states with any number of particles are taken into account within the restrictions due to conservation laws. An essential improvement has been achieved by a careful consideration of three particle reaction channels realized mainly through isobar production. This procedure allows automatically to take into account kinematics of isobar formation but not the dynamics of their subsequent interactions (i.e., it is assumed that isobars decay instantaneously within the nucleus).

Absorption of pions by pairs of nucleons is also taken into account. Time evolution of the system of two interacting nuclei is followed down by a Monte-Carlo technique; local depletion of the nucleon density due to intranuclear collisions and the Pauli principle as well as conservation laws are taken into account in each intranuclear interaction.

The model allows us to consider the emission of nuclear fragments such as ${ }^{2} \mathrm{H},{ }^{3} \mathrm{H},{ }^{3} \mathrm{He}$, and ${ }^{4} \mathrm{He}$. It does it by introducing the dynamical principle of coalescence. ${ }^{24}$ When the cascade interaction stage has been completed, the behavior of an excited residual nucleus is further followed down by a Monte Carlo technique within the framework of the equilibrium statistical approach. The pion component is, of course, insensitive to the coalescence of nucleons in the final state and to the evaporation stage of the interaction. It should be noted, however, that taking into account both effects is very essential for the simulation of experimental selection criteria. A more detailed description of the cascade model used in this work can be found in Refs. 23 and 24. Subsamples of events generated by the model were later on used for the analysis performed in the same way as samples of real events registered and selected in the experiment.

Absolute values of cross sections are satisfactorily reproduced (see Fig. 3). The agreement has been achieved as a consequence of taking into account both the diffuseness of the nuclear boundary and the transparency of the nuclear matter. It should be noted that the distribution of nuclear matter has to be limited to some finite value of the radius $R_{c}$. This cutoff radius is chosen as the distance at which the nuclear density drops down to $1 \%$ of that at the nucleus center. ${ }^{23}$ The reaction cross section $\sigma^{\text {inel }}=\sigma^{\text {geom }} \tau$ turns out to be insensitive to the choice of $R_{c}$ because of the compensating effect of the geometrical size $\sigma^{\text {geom }}=\pi\left(R_{c}^{P}+R_{c}^{T}\right)^{2}$ and the transparency $\tau$.
The Dubna intranuclear cascade model reproduces satisfactorily the dependence of $\left\langle n_{-}\right\rangle / A_{P}$ on $A_{T}$ (Fig. 6); moreover, absolute values of the pion multiplicity for $A_{T} \approx 70$ are close to the experimental ones. A significant discrepancy is seen for the $\mathrm{Pb}$ target. This effect seems to be due to the fact that the standard cascade approach is not able to handle the effects involved in transport through the nucleus of pions with energy close to the $\Delta$ resonance, which leads to an overestimation of the number of $\pi-\mathrm{N}$ interactions and consequently, overestimation of $\left\langle n_{-}\right\rangle$.

The Dubna version of the cascade model applied to the Ar- $\mathrm{KCl}$ collisions at $0.4-1.8 \mathrm{GeV} /$ nucleon leads also to an overestimation of the pion yield; however, the differences are much smaller than in case of the Cugnon cascade version ${ }^{26}$ applied to the analysis of the same data. The latter discrepancies were attributed to the effect of hydrodynamical compression. ${ }^{27}$ This explanation does not seem to work in our case since the projectile size is smaller and incident energy is higher than in the case of the Berkeley data.

It is worthwhile to note that, in spite of the above discussed discrepancy in the absolute yield of pions from the $\mathrm{Pb}$ target, the shapes of the multiplicity distribution are well reproduced by the cascade calculations (see Figs. 7 and 8 ), as well as the decrease of the $\eta_{n}$, with the increasing centrality of collision.

The cascade model allows us to obtain the distribution of the impact parameter $b$ for any given trigger mode and $n_{-} / A_{P}$ value. The average impact parameter is strongly correlated with $n_{-} / A_{P}$. The results of evaluation are shown in Fig. 21. Thus any observed dependence on the trigger mode or $n_{-} / A_{P}$ can be translated into a dependence on the $\langle b\rangle$ value.

Kinematical features of pion emission were also obtained from cascade model calculations. Discrepancies are observed in the case of the $\mathrm{Pb}$ target: Fig. 9 shows that although the decrease of $\langle y\rangle$ with $n_{-} / A_{P}$ observed in the experiment is reproduced, the absolute $\langle y\rangle$ values are underestimated and differ from the experimental data by $\sim 0.1$ rapidity unit. $A$ better agreement with the data is observed in the case of the $\left\langle p_{T}\right\rangle$ dependence on $n_{-} / A_{P}$ [Fig. 12(c)]. The resulting effect is such that the calculated $\left\langle p_{T}\right\rangle-\langle y\rangle$ correlation curve is shifted with respect to experimental data (Fig. 22). The discrepancy may be due to the fact that the participant-participant interactions are not taken into account in the model. The increase of $D_{\cos \theta^{*}}$ with $E^{*} / E_{\max }^{*}$ (Fig. 18) is well reproduced by the model; however, the absolute value $D_{\cos \theta^{*}}$ for the lowest $E^{*}$ values is significantly overestimated. Figure 19(b) demonstrates that the observed correlation between pion and backward protons production is well reproduced by the DICM.

\section{Thermodynamic approach}

Different types of arguments suggest the use of thermodynamic models in the analysis of high energy hadronhadron and nucleus-nucleus collisions. ${ }^{2,29}$ In the models it is assumed that thermic sources (one or more) are 


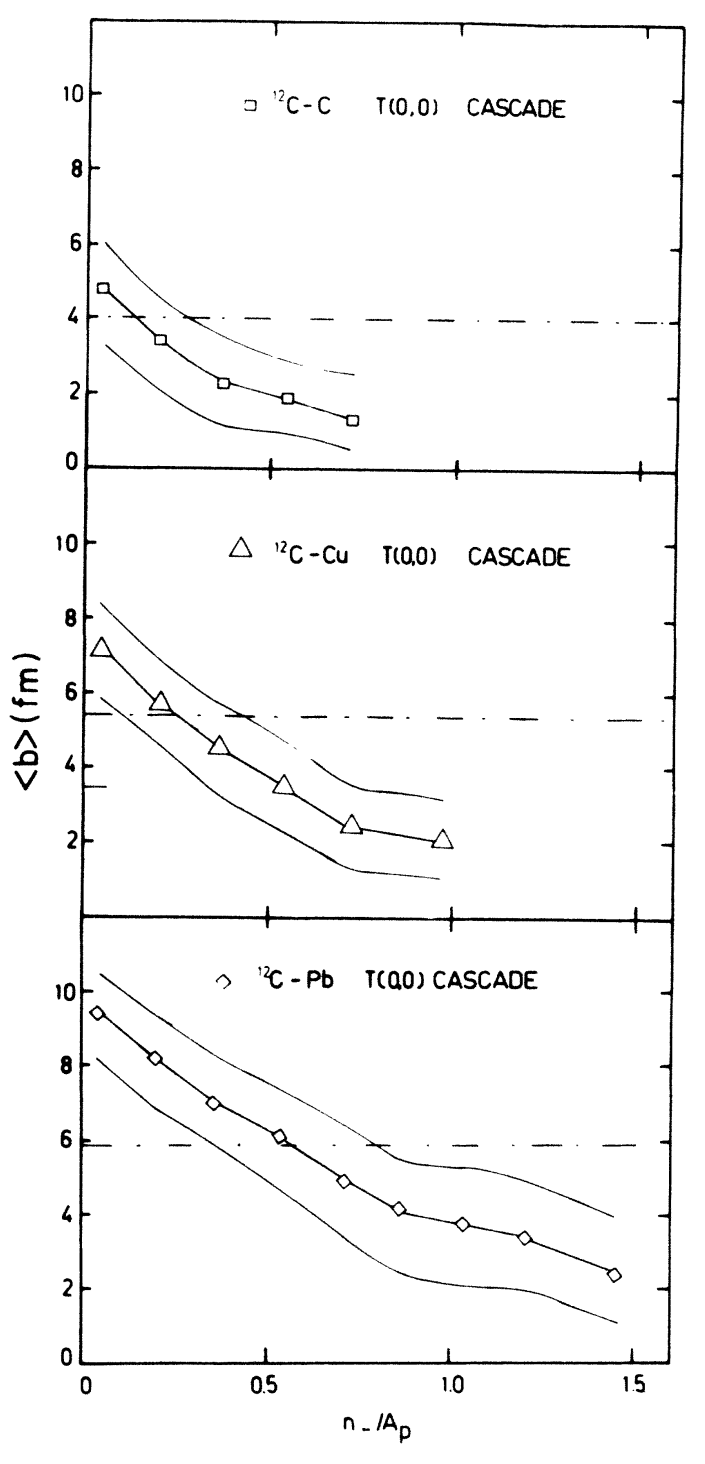

FIG. 21. The average impact parameter values (and the corridor corresponding to $\frac{2}{3}$ area under the $b$ distribution) plotted against $n_{-} / A_{P}$ variable for inelastic collisions of the ${ }^{12} \mathrm{C}$ nuclei with $\mathrm{C}, \mathrm{Cu}$, and $\mathrm{Pb}$ nuclei. The dashed-dotted lines show the $\langle b\rangle$ for all inelastic collisions.

formed in a collision and they move along the direction of the collision axis. The longitudinal velocity of this motion and the temperature are controlled by the energymomentum conservation law. Their values depend also on the assumption on the transparency of nuclear matter. Consequently, the longitudinal motion of the particle sources may be characterized either by a free parameter of the model or by some additional assumptions on the nuclear matter transparency (or its stopping power).

A detailed comparison of data for pions emitted from "average" collisions at our energy with predictions of the thermodynamic-type models ${ }^{30}$ proved that the assumption of a complete stopping of the colliding nuclear matter in its c.m. system is incorrect. The model predicts too high nuclear temperature, too high pion multiplicities, and an-

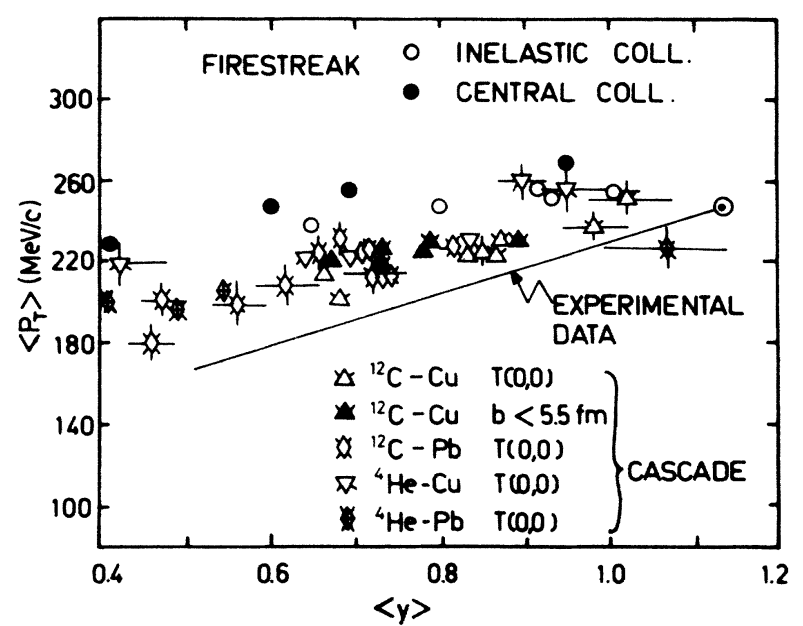

FIG. 22. $\left\langle p_{T}\right\rangle$ plotted against $\langle y\rangle$. Solid line shows the linear fit to experimental data (see Fig. 15). The points show the results of calculations within DICM and within the firestreak model.

gular distributions inconsistent with the observed ones. As an example of such an analysis we show in Fig. 22 a comparison between the experimental data and predictions of the standard firestreak model. ${ }^{31}$ Within the framework of this model central collisions may be defined as those with impact parameter $b<b_{\max }=\pi\left(R_{c}^{T}-R_{c}^{P}\right)^{2}$. Results presented in Fig. 22 are not very sensitive to the degree of the centrality. It is seen that the calculated $\left\langle p_{T}\right\rangle$ values are systematically higher than the experimental ones and that the increase of calculated $\left\langle p_{T}\right\rangle$ values with $\langle y\rangle$ is slower than that obtained for experimental data. This discrepancy shows that the assumption on the fully stopped nuclear matter is not valid. The above conclusion is confirmed by the analysis of the $\left\langle n_{-}\right\rangle$vs $A_{T}$ dependence; the observed increase of $\left\langle n_{-}\right\rangle$with $A_{T}$ is much steeper than that predicted by the model. ${ }^{30}$

In the majority of thermodynamic models, in which a grand canonical ensemble is used, ${ }^{32}$ the multiplicity distribution of pions emitted directly from a single thermic source is of the Poisson-type. ${ }^{29}$ The distribution is expected to be broader if more than one fireball is produced in the collision, and if an averaging over the impact parameter, leading to the sources of different temperature, takes place. The experimental distributions for central collisions are, however, narrower than the Poisson distribution (Fig. 7), in contradiction with standard thermodynamic model predictions. Attempts to reproduce our data by the model with various assumptions about stopping power of nuclear matter were undertaken in Ref. 30; none turned out to be successful.

Our further analysis is carried out rather "in a general spirit" of the thermodynamic approach than within a particular model version. Pion samples analyzed in the previous sections of this paper did not show isotropy of their emission in any conceivable reference system with one exception-high $n_{-} / A_{P}$ subsamples of inelastic ${ }^{4} \mathrm{He}-\mathrm{Li}$ collisions (Fig. 17). However, this may be considered as a 
manifestation of the features of $\mathrm{N}-\mathrm{N}$ interactions. Moreover, as shown in Fig. 16, in the case of $A_{P}<A_{T}$ collisions it has not been possible to find a reference system in which pion emission would reveal backward-forward symmetry. The only pion sample in which the emission isotropy has been observed is the sample of pions produced in " $\Lambda$ out" events of central ${ }^{12} \mathrm{C}-\mathrm{C},{ }^{12} \mathrm{C}-\mathrm{Ne}$, and ${ }^{16} \mathrm{O}-\mathrm{Ne}$ collisions (see Sec. VI). One should note that $\Lambda$ hyperons from these central collisions are also produced isotropically in the N-N c.m. system. ${ }^{13}$

The average temperature $T_{0}$ of thermic sources of particles can be obtained from transverse momentum data, assuming that the particles are emitted directly from the sources and using the following formula:

$$
\left\langle p_{T}\right\rangle=\left(\frac{\pi m T_{0}}{2}\right)^{1 / 2} K_{5 / 2}\left(\frac{m}{T_{0}}\right) / K_{2}\left(\frac{m}{T_{0}}\right),
$$

where $K_{\alpha}(x)$ are the so-called McDonald functions. The formula was derived ${ }^{28}$ within the Boltzmann approximation and under the assumption that the only collective motion is the longitudinal motion. The obtained $T_{0}$ value for $\Lambda$ 's produced in central ${ }^{12} \mathrm{C}-\mathrm{C},{ }^{12} \mathrm{C}-\mathrm{Ne}$, and ${ }^{16} \mathrm{O}-\mathrm{Ne}$ collisions is $T_{0}=(150 \pm 19) \mathrm{MeV}$ and for pions produced in " $\Lambda$ out" events it is $T_{0}=(114 \pm 11) \mathrm{MeV} \cdot{ }^{33}$ These results are plotted in Fig. 23, where our "temperature data" and those from other experiments (nucleus-nucleus collisions $^{34}$ and $\mathrm{N}-\mathrm{N}$ collisions ${ }^{19}$ ) are plotted against the beam kinetic energy $E_{\text {beam }}^{*}$. The dotted and dashed lines presenting N-N data correspond to $T_{0}$ values calculated from the $\left\langle p_{T}\right\rangle$ and from the $\left[d^{3} \sigma / d p^{3}\left(\theta^{*}=90^{\circ}\right)\right.$ $\left.\sim \exp \left(-E^{*} / T_{0}\right)\right]$ fits, respectively. It is seen that the highest temperatures are obtained for the first two samples of events. The results may suggest that single hot sources stopped in the c.m. system are formed in central collisions of roughly symmetric light ions, in which $\Lambda$ hyperons are produced beyond kinematical $\mathrm{N}-\mathrm{N}$ limits. These observations are consistent with expectations derived from the thermodynamic approach. Within this approach $\Lambda$ 's, of course, are produced more abundantly in high $T_{0}$ events, therefore $\Lambda$ hyperons and especially $\Lambda$ 's produced beyond kinematical limits give a signature of events in which full stopping of nuclear matter and consequently high $T_{0}$ are achieved. The experimental $T_{0}$ values are consistent with the results of calculations performed assuming the full thermalization of nuclear matter, ${ }^{35}$ shown as solid lines in Fig. 23.

\section{CONCLUSIONS}

This paper presents the data on $\pi^{-}$meson production in inelastic and central collisions of ${ }^{4} \mathrm{He},{ }^{12} \mathrm{C},{ }^{16} \mathrm{O}$, and ${ }^{20} \mathrm{Ne}$ nuclei accelerated up to $4.5 \mathrm{GeV} / c$ momentum per incident nucleon with various nuclear targets, ranging from $\mathrm{Li}$ to $\mathrm{Pb}$. A detailed analysis of the influence of the central trigger on observed multiplicity distributions and kinematical characteristics was performed.

The angular constraints imposed on projectile fragments registered by the triggering system deplete strongly the low multiplicity region of the partial cross sections, whereas the cross sections for the high multiplicity region are left unchanged. The latter statement concerns trigger

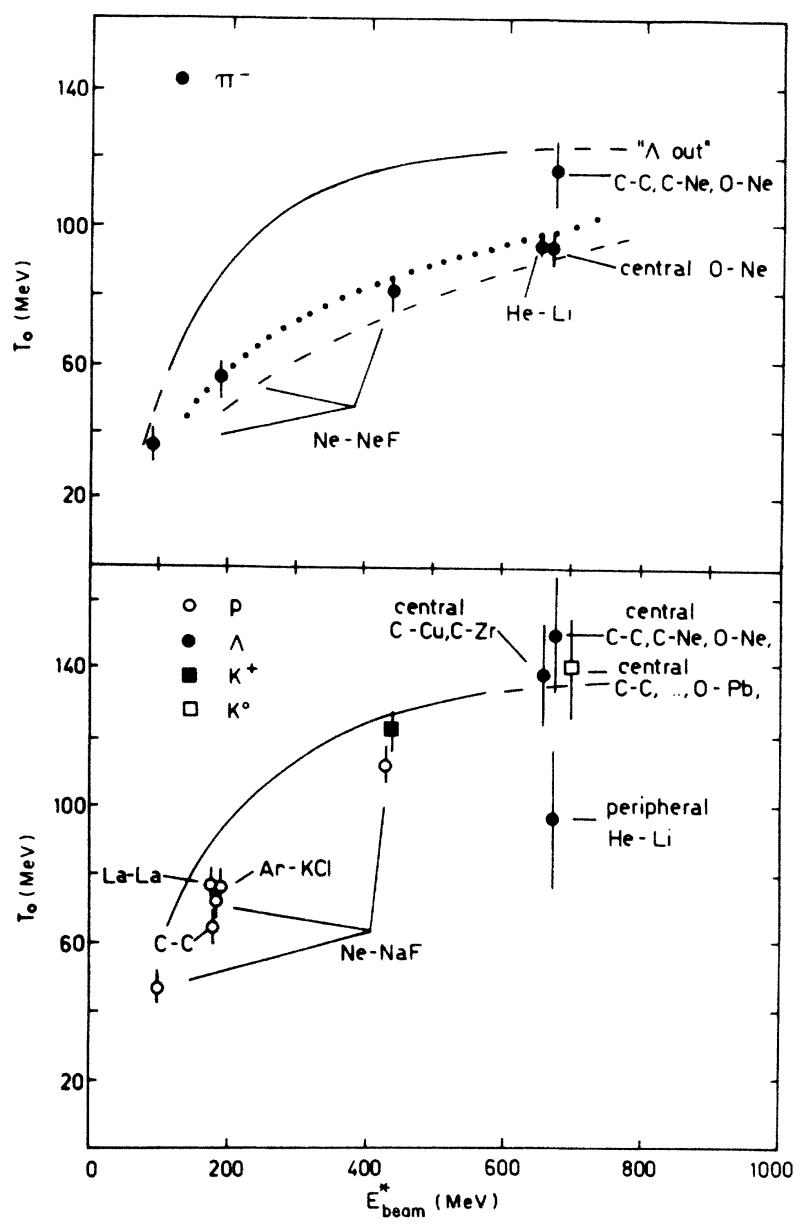

FIG. 23. The source temperature $T_{0}$ plotted against the beam kinetic energy $E_{\text {beam }}^{*}$ (see the text).

veto angles up to $\sim 3^{\circ}$ since a further increase of the veto angle superimposes extra conditions (high inelasticity) on central collisions.

For a given pair of colliding nuclei the spectra of pion rapidities, transverse momenta, and emission angles in the $\mathrm{N}-\mathrm{N}$ c.m. system depend on the collision multiplicity but they are independent of the central trigger for a fixed multiplicity. Analysis of $y$ and $p_{T}$ spectra shows that the data for a given $A_{T}$ scale in the variable $n_{-} / A_{P}$. This variable may be considered as a measure of the thickness of the interacting layer of target nucleus and consequently of the impact parameter. The calculations carried out within the framework of the intranuclear cascade model show that by fixing the $n_{-} / A_{P}$ value the impact parameter interval is limited to the width of about $2 \mathrm{fm}$.

The rapidity and transverse momentum data scale in the $\langle y\rangle$ variable for subsamples of events characterized by given masses of colliding nuclei, the trigger mode, and multiplicity of pions. The observation suggests that $\langle y\rangle$ may be treated as a "universal" (independent of $A_{T}$ ) measure of an average thickness of the target nuclear matter involved in the collision provided that $A_{P}<A_{T}$. 
One should keep in mind that although the rapidity distributions are roughly symmetric with respect to the $y=\langle y\rangle$ line, significant asymmetry of $y-p_{T}$ plots is observed even in the case of subsamples of central events which are characterized by the small dispersion of their impact parameter.

The comparison of the experimental multiplicity data with predictions of theoretical models shows that the experimental average multiplicities are only slightly higher than those predicted as the maximum values by the nocascading model. Taking into account cascading processes within the Dubna intranuclear cascade model leads to an overestimation (by about $45 \%$ for ${ }^{4} \mathrm{He}$ and $25 \%$ for ${ }^{12} \mathrm{C}$ projectiles) of the pion multiplicity from our heaviest target, $\mathrm{Pb}$. The analysis of kinematical features of pions demonstrates, however, that the inclusion of cascading processes is necessary for the description of the data. Although the cascade model reproduces satisfactorily the main features of the kinematical data, some quantitative discrepancies are observed.

A complete disagreement (by about $100 \%$ ) is seen in the case of the comparison of multiplicity data with predictions of thermodynamic models assuming the full stop- ping of nuclear matter. This observation as well as the detailed study of kinematical features of pion production indicate that full stopping of the colliding layers of the nuclear matter does not take place even in high multiplicity central collisions with the $\mathrm{Pb}$ target.

Thus we conclude that the main body of the pion production data can be understood within the cascade model. However, analysis of pions associated with $\Lambda$ production revealed an observation which seems to be unexplainable within such an approach.

The sample of central collisions of symmetric $\left(A_{P} \approx A_{T}\right)$ ions with an additional criterion of a $\Lambda$ produced beyond the $\mathbf{N}-\mathbf{N}$ kinematical limits was selected $\left(\sigma=10^{-3} \sigma^{\text {inel }}\right)$. The angular distributions of pion and energy yields for such events are consistent with the isotropy of the pion emission in the N-N c.m. system in contrast to pion distributions from other samples of events. Average transverse momentum of these pions is higher than that of pions from other samples of events.

Three of the authors (M.G., E.S., and R.S. from the University of Warsaw) acknowledge financial support from the Ministry of Science and Higher Education, Contract No. MRI. 5.
1S. Nagamiya and M. Gyulassy, Adv. Nucl. Phys. 13, 201 (1984), and references therein.

${ }^{2}$ S. Fredriksson et al., Royal Institute of Technology Report TRITA-TFY-84-06, 1984, and reference therein.

${ }^{3}$ M. Akhababian et al., Z. Phys. C 16, 307 (1983).

${ }^{4}$ R. Stock et al., CERN Report CERN/PSCC/80-129, 1980; C.

R. Gruhn et al., CERN Report CERN/SPSC/84-13, 1984;

H. Gordon et al., CERN Report CERN/SPSC/84-43, 1984;

N. Armeise et al., CERN Report CERN/SPSC/84-69, 1984; K. B. Bhalla et al., CERN Report CERN/SPSC/84-27, 1984.

${ }^{5}$ Quark Matter Formation and Heavy Ion Collisions, edited by M. Jacob and J. Tran Thanh Van, Phys. Rep. 88, 321 (1982), and references therein.

${ }^{6} \mathrm{M}$. Gyulassy, in Proceedings of the International Conference on High Energy Nuclear Physics, Balatonfüred, Hungary 1983, edited by J. Erö, p. 489.

${ }^{7}$ K. K. Gudima and V. D. Toneev, Yad. Fiz. 31, 1455 (1980).

${ }^{8}$ V. Aksinenko et al., Nucl. Phys. A324, 266 (1978).

${ }^{9}$ V. Aksinenko et al., Nucl. Phys. A348, 518 (1980).

${ }^{10}$ A. Abdurakhimov et al., Nucl. Phys. A362, 376 (1981).

${ }^{11}$ M. Anikina et al., Z. Phys. C 9, 105 (1981).

${ }^{12}$ M. Anikina et al., Phys. Rev. Lett. 50, 1971 (1983).

${ }^{13}$ M. Anikina et al., Z. Phys. C 25, 1 (1984).

${ }^{14} \mathrm{M}$. Anikina et al., Joint Institute of Nuclear Research Report E1-84-785, 1984.

${ }^{15}$ M. Gazdzicki et al., Joint Institute of Nuclear Research Report E1-84-444, 1984.

${ }^{16}$ M. Anikina et al., Yad. Fiz. 40, 489 (1984).

${ }^{17}$ H. L. Bradt and B. Peters, Phys. Rev. 77, 54 (1950).

${ }^{18}$ E. J. Moniz et al., Phys. Rev. Lett. 26, 445 (1971).

${ }^{19}$ A. Abdivaliev et al., Joint Institute of Nuclear Research Report P1-82-507, 1982. 20(a) R. Brockmann et al., Phys. Rev. Lett. 53, 2012 (1984); (b) J. W. Harris et al., ibid. 47, 229 (1981).

${ }^{21}$ M. Anikina et al., Joint Institute of Nuclear Research Report E1-84-376, 1984.

${ }^{22}$ M. Anikina et al., Z. Phys. C 18, 109 (1983).

${ }^{23}$ K. K. Gudima and V. D. Toneev, Yad. Fiz. 27, 658 (1978).

${ }^{24}$ V. D. Toneev and K. K. Gudima, Nucl. Phys. A400, 173 (1983).

${ }^{25}$ V. S. Barashenkov and V. D. Toneev, Interactions of High Energy Particles and Atomic Nuclei with Nuclei (Atomizdat, Moscow, 1972).

26J. Cugnon, Phys. Rev. C 22, 885 (1980).

${ }^{27}$ R. Stock et al., Phys. Rev. Lett. 49, 1236 (1982).

${ }^{28}$ R. Hagedorn, CERN Report 71-12, 1971.

${ }^{29}$ S. Das Gupta and A. Z. Mekjian, Phys. Rep. 72, 131 (1981).

${ }^{30}$ M. Gaździcki and St. Mrówczyński, Phys. Rev. C 30, 388 (1984).

${ }^{31}$ J. Gosset, J. I. Kapusta, and G. D. Westfall, Phys. Rev. C 18, 844 (1978).

${ }^{32}$ Discussion of the multiplicity distributions in so-called pressure ensamble can be found in St. Mrówczyński, Joint Institute of Nuclear Research Report E2-84-193, 1984.

${ }^{33}$ It should be noted that temperature data obtained for pions are expected to be underestimated due to a significant contribution of pions produced via $\Delta$ resonances decay.

${ }^{34}$ S. Nagamiya, Nucl. Phys. A418, 239 (1984), and references therein; to calculate $T_{0}$ values from the data we used the $\left[d^{3} \sigma / d p^{3}\left(\theta^{*}=90^{\circ}\right) \sim \exp \left(-E^{*} / T_{0}\right)\right]$ fit, since the often used parameter $E_{0}$ derived from the $\left[E d^{3} \sigma / d p^{3}\left(\theta^{*}=90^{\circ}\right)\right.$ $\left.\sim \exp \left(-E^{*} / E_{0}\right)\right]$ fit cannot be interpreted as the temperature (generally $E_{0}>T_{0}$ ).

${ }^{35}$ R. Hagedorn and J. Rafelski, Phys. Lett. 97B, 136 (1980). 